TRANSACTIONS OF THE

AMERICAN MATHEMATICAL SOCIETY

Volume 355, Number 7, Pages 2633-2649

S 0002-9947(03)03271-9

Article electronically published on February 27, 2003

\title{
THE ALMOST-DISJOINTNESS NUMBER MAY HAVE COUNTABLE COFINALITY
}

\author{
JÖRG BRENDLE
}

Abstract. We show that it is consistent for the almost-disjointness number $\mathfrak{a}$ to have countable cofinality. For example, it may be equal to $\aleph_{\omega}$.

\section{INTRODUCTION}

Cardinal invariants of the continuum, that is, cardinal numbers between $\aleph_{1}$ and $\mathfrak{c}$ (the size of the continuum) which are defined as the smallest size of a family of real numbers with a certain combinatorial property, play an increasingly important role in modern set theory. Equalities and inequalities between cardinal invariants have many connections with problems arising naturally in general topology, real analysis and algebra, and, from a purely set-theoretic point of view, there is a deep interplay with forcing theory, in particular in the light of the search for new iteration techniques.

One of the most basic questions about cardinal invariants is which values they can assume, and, for almost all cardinals, it is known that any regular value is possible 1 Furthermore, most cardinals can either be shown to be regular in $Z F C$ or they are equal to $\mathfrak{c}$ in the random real model, in the Cohen real model. ${ }^{2}$ or even in both, so that they can be consistently singular of uncountable cofinality. Notable exceptions are the splitting number $\mathfrak{s}$ of which it is still unknown whether it may be singular $[\mathrm{V}]$ and the almost-disjointness number $\mathfrak{a}$, which has recently been shown to be consistently singular of uncountable cofinality by Shelah S2 3 Things get trickier when one considers singular cardinals of countable cofinality. In fact, by far most of the cardinals, even those singular in the Cohen or random models, can be shown to have uncountable cofinality in $Z F C$ Exceptions are

- Shelah [S1] has proved that the covering number of the null ideal may have countable cofinality,

- the almost-disjointness number $\mathfrak{a}$ dealt with below,

Received by the editors October 3, 2001.

2000 Mathematics Subject Classification. Primary 03E17; Secondary 03E35.

Key words and phrases. Maximal almost-disjoint families, almost-disjointness number, iterated forcing.

Supported by Grant-in-Aid for Scientific Research (C)(2)12640124, Japan Society for the Promotion of Science.

${ }^{1}$ In fact, most of the cardinals that have been studied are equal to $\mathfrak{c}$ under Martin's axiom $M A$.

${ }^{2}$ The models obtained by adding $\kappa=\kappa^{\omega}$ many random or Cohen reals.

${ }^{3}$ Cardinals relevant for this paper will be defined below. Also see $[\mathrm{vD}],[\mathrm{V}]$, or [B] .

${ }^{4}$ This is also true for $\mathfrak{s}$. 
- it is unknown whether the reaping number $\mathfrak{r}[\mathrm{M}$, Problem 3.4] or the independence number $\mathfrak{i}$ can have countable cofinality

Here we show

Main Theorem. Assume $C H$ and let $\lambda$ be a singular cardinal of countable cofinality. Then there is a forcing extension satisfying $\mathfrak{a}=\lambda$. In particular, $\mathfrak{a}=\aleph_{\omega}$ is consistent.

We give a brief outline of the proof. It is well known that, assuming $C H$, one can force a mad family of size $\aleph_{\omega}$ such that, by a standard isomorphism-of-names argument, there is no mad family of size $\aleph_{n}$ for $n \geq 2$ in the generic extension [H1]. However, mad families of size $\aleph_{1}$ may survive the forcing. The simplest way to get rid of small mad families is by iteratively adding dominating reals, say for $\aleph_{2}$ steps. If this is done in such a way that every dominating real is dominating only over a fragment of the mad family of size $\aleph_{\omega}$ added in the initial step, the latter will survive. Yet, if the dominating reals are added in a standard way, the forcing will lose most of its homogeneity, the isomorphism-of-names argument will cease to work, and there may be a mad family of size $\aleph_{2}$ instead. This is where Shelah's recent technique of iteration along templates [S2 comes in 6 It provides for a way of adjoining dominating reals with a forcing having enough local homogeneity. However, since isomorphism-of-names arguments require $\mathrm{CH}$ in the ground model, we need to describe the two-step extension sketched above in one step and incorporate the forcing adding the mad family of size $\aleph_{\omega}$ into the template framework. This accounts for some of the technical difficulties described below.

Apart from proving the Main Theorem, we also present a new, more axiomatic, treatment of the template framework (Section 11). While Shelah S2 defines the template via two procedures building more complicated sets (with larger depth) from simpler ones, we only require that the template is a family of subsets of the linear order underlying the iteration satisfying several axioms, most notably well-foundedness. We think this approach is more lucid, apart from providing for a simpler definition of the iteration. There is a price one has to pay for that, however: the Completeness Lemma 1.1 showing that we are indeed dealing with an iteration, requires some additional work. Of course, our general approach can also be used to prove Shelah's original results [S2] (see [B] $)$ and, since there is no need to add a mad family in this case, definitions will be simpler than in Section 1

In Section 2 we describe the template used for the proof of the Main Theorem, and in Section 3 we provide the isomorphism-of-names argument needed to complete the proof.

The template framework developed in Section 1 for Hechler forcing can in fact be used to handle a large class of easily definable ccc forcing notions (see $\mathrm{Br}$, Section 4] for a few examples). Replacing the forcing generically adjoining a mad family by an appropriate relative, one can get analogous results for relatives of $\mathfrak{a}$, e.g., for $\mathfrak{a}_{\mathfrak{s}}$, the size of the smallest mad family of partial functions from $\omega$ to $\omega$. So, for example, $\mathfrak{a}_{\mathfrak{s}}=\aleph_{\omega}$ is consistent, and so is $\mathfrak{a}=\aleph_{1}<\mathfrak{a}_{\mathfrak{s}}=\aleph_{\omega}$. For the latter

\footnotetext{
${ }^{5} \mathrm{As}$ for maximal almost-disjoint families, it is rather easy to force a maximal independent family of size, say, $\aleph_{\omega}$. Both $\mathfrak{r}$ and $\mathfrak{i}$ are equal to $\mathfrak{c}$, and thus possibly singular, in the Cohen and random models.

${ }^{6}$ As mentioned above, Shelah showed among other things that $\mathfrak{a}$ could be singular. In his models, $\mathfrak{a}$ is equal to $\mathfrak{c}$ and therefore has uncountable cofinality.
} 
result, one needs to replace Hechler forcing by eventually-different-reals forcing in the framework of Section 1 (cf. [Br Section 4]).

Let us briefly recall the main notions relevant for this paper. Two infinite subsets $A$ and $B$ of $\omega$ are called almost-disjoint if their intersection is finite. $\mathcal{A} \subseteq[\omega]^{\omega}$ is an almost-disjoint family if its members are pairwise almost disjoint. $\mathcal{A}$ is a mad family (maximal almost-disjoint family) if it is maximal with respect to being an almost-disjoint family, i.e., for every $B \in[\omega]^{\omega}$ there is $A \in \mathcal{A}$ such that $A \cap B$ is infinite. The almost-disjointness number $\mathfrak{a}$ is the size of the least infinite mad family. For functions $f, g \in \omega^{\omega}$, we say that $g$ eventually dominates $f$ (and write $\left.f \leq^{*} g\right)$ if the set $\{n ; f(n)>g(n)\}$ is finite. The unbounding number $\mathfrak{b}$ is the cardinality of the smallest unbounded family in the structure $\left(\omega^{\omega}, \leq^{*}\right)$, that is, the size of the smallest $\mathcal{F} \subseteq \omega^{\omega}$ such that for all $g \in \omega^{\omega}$ there is $f \in \mathcal{F}$ with $f \mathbb{Z}^{*} g$. The dominating number $\mathfrak{d}$ is the size of the least cofinal family in $\left(\omega^{\omega}, \leq^{*}\right)$. It is well known and easy to see that $\mathfrak{b} \leq \mathfrak{d}$ and $\mathfrak{b} \leq \mathfrak{a}$ in $Z F C$ vD.

Hechler forcing [H2] $\mathbb{D}$ (see also [BJ]) consists of pairs $(s, f)$ where $s \in \omega^{<\omega}$, $f \in \omega^{\omega}$ and $s \subseteq f$, ordered by $(t, g) \leq(s, f)$ if $t \supseteq s$, and $g \geq f$ everywhere. It generically adds a dominating real, that is, a real that eventually dominates all ground model reals. It is this forcing adjoining a dominating real which we shall use in the template framework sketched above.

Our notation is standard. For cardinal invariants of the continuum, we refer to $[\mathrm{vD}], \mathrm{V}]$ or $[\mathrm{Bl}]$. For forcing theory, in particular for forcing related to cardinal invariants of the continuum, see [BJ].

I thank Juris Steprāns for pointing out a flaw in an earlier version of this work.

\section{Templates And iterations}

The most useful definition of a template seems to be (see also $[\mathrm{Br}]$ ) the following.

Definition (Template). A template is a pair $(L, \mathcal{I})$ such that $(L, \leq)$ is a linear order and $\mathcal{I} \subseteq \mathcal{P}(L)$ is a family of subsets of $L$ satisfying

(1) $\emptyset, L \in \mathcal{I}$,

(2) $\mathcal{I}$ is closed under finite unions and intersections,

(3) if $y<x$ belong to $L$, then there is $A \in \mathcal{I} \cap \mathcal{P}\left(L_{x}\right)$ such that $y \in A$,

(4) if $A \in \mathcal{I}$ and $x \in L \backslash A$, then $A \cap L_{x} \in \mathcal{I}$,

(5) $\mathcal{I}$ is well-founded, i.e., there is a function $\mathrm{Dp}=\mathrm{Dp}_{\mathcal{I}}: \mathcal{I} \rightarrow$ On, called depth, recursively defined by $\operatorname{Dp}(\emptyset)=0$ and $\operatorname{Dp}(A)=\sup \{\operatorname{Dp}(B)+1 ; B \in \mathcal{I}$ and $B \subset A\}$ for $A \in \mathcal{I} \backslash\{\emptyset\}$.

Here, $L_{x}=\{y \in L ; y<x\}$ is the restriction of $L$ to $x$. If $A \subseteq L$, we define $\mathcal{I} \mid A=\{B \cap A ; B \in \mathcal{I}\}$, the trace of $\mathcal{I}$ on $A .(A, \mathcal{I} \mid A)$ is also a template.

Since $\mathcal{I}$ is closed under finite intersections, if $A \in \mathcal{I}$, then $\mathcal{I}\lceil A=\{B \in \mathcal{I} ; B \subseteq$ $A\}$.

In our context, we need to slightly revise this definition because we will not only have "iteration coordinates" $L_{\text {Hech }}$ used for adjoining Hechler generics but also "product coordinates" $L_{\mathrm{mad}}$ used for adding a mad family. Since the former should be generic over some, but not all, of the latter, we need to incorporate them into the same template framework $L$, and some of the above clauses should be true for all of $L$, while others need to be satisfied only for members of $L_{\mathrm{Hech}}$. 
Accordingly, let $L_{\mathrm{Hech}}$ and $L_{\mathrm{mad}}$ be disjoint sets, put $L=L_{\mathrm{Hech}} \cup L_{\mathrm{mad}}$, and assume $L$ is equipped with a linear order. Further suppose $\mathcal{I} \subseteq \mathcal{P}(L)$ satisfies, in addition to (1) and (2) above, the following clauses:

$\left(3^{\prime}\right)$ if $x \in L_{\text {Hech }}$ and $y \in L_{x}$, then there is $A \in \mathcal{I} \cap \mathcal{P}\left(L_{x}\right)$ such that $y \in A$;

$\left(4^{\prime}\right)$ if $A \in \mathcal{I}$ and $x \in L_{\mathrm{Hech}} \backslash A$, then $A \cap L_{x} \in \mathcal{I}$;

$\left(5^{\prime}\right)$ the trace $\mathcal{I}\left\lceil L_{\mathrm{Hech}}=\left\{A \cap L_{\mathrm{Hech}} ; A \in \mathcal{I}\right\}\right.$ is well-founded,

as well as

(6) if $A \in \mathcal{I}$ and $x \in A$, then $L_{x} \cap L_{\text {mad }} \subseteq A$.

This is the definition of "template" we shall work with for the remainder of the paper. Notice that $\left(5^{\prime}\right)$ means in particular that the depth function Dp depends only on the $L_{\text {Hech }}$-part, i.e., $\operatorname{Dp}(A)=0$ iff $A \subseteq L_{\text {mad }}$ and, recursively, $\operatorname{Dp}(A)=$ $\sup \left\{\operatorname{Dp}(B)+1 ; B \in \mathcal{I}\right.$ and $\left.B \cap L_{\text {Hech }} \subset A \cap L_{\text {Hech }}\right\}$.

(6) is a closure condition for the $L_{\mathrm{mad}}$-part which is needed to make the proof of Main Lemma 1.1 below go through. More generally, we say that $A \subseteq L$ is closed if $A$ satisfies (6). (So $\mathcal{I}$ consists only of closed sets.) For arbitrary $A \subseteq L$, we then define its closure $\operatorname{cl}(A)$ by $\operatorname{cl}(A)=A \cup \bigcup_{x \in A}\left(L_{x} \cap L_{\text {mad }}\right)$. Thus cl $(A)$ is the smallest closed set containing $A$ and $\operatorname{cl}(\mathrm{cl}(A))=\mathrm{cl}(A)$.

The basic idea for the following, attempted, definition comes from $\mathrm{S} 2$. It is modified, however, due to our axiomatic treatment of the concept of "template" (see also $[\mathrm{Br}]$ ) and because of the inclusion of $L_{\mathrm{mad}}$.

Definition (Iterating Hechler forcing and adding a mad family along a template). Assume $(L, \mathcal{I})$ is as above. We define, for $A \in \mathcal{I}$, by recursion on $\operatorname{Dp}(A)$, the partial order (p.o.) $\mathbb{P}\lceil A$ (more explicitly, we define $\mathbb{P} \uparrow(A, \mathcal{I})$, but we shall drop the reference to $\mathcal{I}$ in case there is no ambiguity).

- $\operatorname{Dp}(A)=0$. This means $A \subseteq L_{\mathrm{mad}}$. $\mathbb{P} \nmid A$ consists of all finite partial functions $p$ with domain contained in $A$ and such that $p(z) \in 2^{n}$ for all $z \in \operatorname{dom}(p)$ for some $n=n^{p} \in \omega$. The ordering on $\mathbb{P} \nmid A$ is given by: $q \leq_{\mathbb{P} \nmid A} p$ if $\operatorname{dom}(q) \supseteq \operatorname{dom}(p)$ and

- $n^{q} \geq n^{p}, p(z) \subseteq q(z)$ for all $z \in \operatorname{dom}(p),|\{z \in \operatorname{dom}(p) ; q(z)(i)=1\}| \leq$ 1 for all $i \in n^{q} \backslash n^{p}$.

- $\operatorname{Dp}(A)>0 . \quad \mathbb{P}\lceil A$ consists of all finite partial functions $p$ with domain contained in $A$ and such that

- there is $n=n^{p} \in \omega$ with $p(z) \in 2^{n}$ for all $z \in \operatorname{dom}(p) \cap L_{\text {mad }}$;

- letting $x=\max \left(\operatorname{dom}(p) \cap L_{\text {Hech }}\right)$, there is $B \in \mathcal{I} \cap \mathcal{P}\left(A \cap L_{x}\right)$ (so $\operatorname{Dp}(B)<\operatorname{Dp}(A))$ such that $p\left\lceil\left(A \cap L_{x}\right) \in \mathbb{P}\left\lceil B\right.\right.$ and $p(x)=\left(s_{x}^{p}, \dot{f}_{x}^{p}\right)$ where $s_{x}^{p} \in \omega^{<\omega}$ and $\dot{f}_{x}^{p}$ is a $\mathbb{P}\left\lceil B\right.$-name for an element of $\omega^{\omega}$ such that $p \uparrow\left(A \cap L_{x}\right) \Vdash_{\mathbb{P} \uparrow B}$ " $s_{x}^{p} \subseteq \dot{f}_{x}^{p}$ " (this means $p(x)$ is a $\mathbb{P}\lceil B$-namष for a condition in Hechler forcing $\dot{\mathbb{D}})$.

The ordering on $\mathbb{P} \nmid A$ is given by: $q \leq \mathbb{P} \nmid A$ if $\operatorname{dom}(q) \supseteq \operatorname{dom}(p)$ and

$-n^{q} \geq n^{p}, p(z) \subseteq q(z)$ for all $z \in \operatorname{dom}(p) \cap L_{\mathrm{mad}}, \mid\{z \in \operatorname{dom}(p) \cap$ $\left.L_{\text {mad }} q q(z)(i)=1\right\} \mid \leq 1$ for all $i \in n^{q} \backslash n^{p}$ (this guarantees that the reals added in coordinates from $L_{\mathrm{mad}}$ are characteristic functions of an almost-disjoint family), as well as

\footnotetext{
${ }^{7}$ Note, however, that the first coordinate $s_{x}^{p}$ of the condition $p(x)$ is not a name.
} 
- either $y=\max \left(\operatorname{dom}(q) \cap L_{\text {Hech }}\right)>x=\max \left(\operatorname{dom}(p) \cap L_{\text {Hech }}\right)$ and there is $B \in \mathcal{I} \cap \mathcal{P}\left(A \cap L_{y}\right)$ such that $p\left\lceil\left(A \cap L_{y}\right), q \uparrow\left(A \cap L_{y}\right) \in \mathbb{P}\lceil B\right.$ and $q \uparrow\left(A \cap L_{y}\right) \leq_{\mathbb{P} \uparrow B} p \uparrow\left(A \cap L_{y}\right)$,

- or $x=\max \left(\operatorname{dom}(q) \cap L_{\mathrm{Hech}}\right)=\max \left(\operatorname{dom}(p) \cap L_{\mathrm{Hech}}\right)$ and there is $B \in \mathcal{I} \cap \mathcal{P}\left(A \cap L_{x}\right)$ such that $p\left\lceil\left(A \cap L_{x}\right), q \uparrow\left(A \cap L_{x}\right) \in \mathbb{P}\lceil B, q \uparrow(A \cap\right.$ $\left.L_{x}\right) \leq_{\mathbb{P} \uparrow B} p\left\lceil\left(A \cap L_{x}\right), \dot{f}_{x}^{p}, \dot{f}_{x}^{q}\right.$ are $\mathbb{P}\left\lceil B\right.$-names, $s_{x}^{p} \subseteq s_{x}^{q}$, and $q \uparrow(A \cap$ $\left.L_{x}\right) \Vdash_{\mathbb{P} \nmid B}$ " $\dot{f}_{x}^{p}(n) \leq \dot{f}_{x}^{q}(n)$ for all $n$ " (the last two clauses mean that $\left.q \uparrow\left(A \cap L_{x}\right) \Vdash_{\mathbb{P} \uparrow B} " q(x) \leq_{\dot{\mathbb{D}}} p(x) "\right)$.

We have not argued yet that this recursive definition works at all. The point is this requires that all $\mathbb{P}\lceil A$ 's be transitive, which is not trivial because the sets $B \in \mathcal{I}$ witnessing that $q \leq p$ may depend on the pair $(p, q)$. Therefore, to prove transitivity, we need to show that the $\mathbb{P}\lceil B$ completely embed one into the other. This will be done in Main Lemma 1.1 below.

Note that, once this is achieved, $\mathbb{P}\lceil(A, \mathcal{I})=\mathbb{P}\lceil(A, \mathcal{I}\lceil A)$ is immediate for $A \in \mathcal{I}$. Of course, the above recursion also defines $\mathbb{P}\lceil A=\mathbb{P}\lceil(A, \mathcal{I}\lceil A)$ for arbitrary $A \subseteq L$.

If $A, B \in \mathcal{I}, A \subset B$, then $\mathbb{P}\lceil A \subset \mathbb{P}\lceil B$ is immediate from the definition (because $\mathcal{I} \nmid A \subset \mathcal{I}\lceil B$ in this case). This is much less clear if one of $A$ or $B$ does not belong to $\mathcal{I}$. Neither is it clear whether $\mathbb{P}\lceil A<0 \mathbb{P}\lceil B$, the most basic property the above recursive definition must satisfy to make it an iteration, even in case both $A$ and $B$ come from $\mathcal{I}$. This issue is addressed by the following crucial lemma.

Main Lemma 1.1 (Completeness of embeddings). Let $B \in \mathcal{I}$ and $A \subset B$ be closed. Then $\mathbb{P}\lceil B$ is a partial order, $\mathbb{P}\lceil A \subset \mathbb{P}\lceil B$ and even $\mathbb{P}\lceil A<0 \mathbb{P}\lceil B$. More explicitly, any $p \in \mathbb{P} \mid B$ has a canonical reduction $p_{0}=p_{0}(p, A, B) \in \mathbb{P} \nmid A$ such that

(i) $\operatorname{dom}\left(p_{0}\right)=\operatorname{dom}(p) \cap A$;

(ii) $s_{x}^{p_{0}}=s_{x}^{p}$ for all $x \in \operatorname{dom}\left(p_{0}\right) \cap L_{\text {Hech }}$ and $p_{0}(x)=p(x)$ for all $x \in \operatorname{dom}\left(p_{0}\right) \cap$ $L_{\text {mad }}$ (in particular, $n^{p_{0}}=n^{p}$ ),

and such that, whenever $D \in \mathcal{I}, B, C \subseteq D, C$ closed, $C \cap B=A$, and $q_{0} \in \mathbb{P}\lceil C$ extends $p_{0}$, then there is $q \in \mathbb{P}\left\lceil D\right.$ extending both $q_{0}$ and $p$.

Note that we do not require $p \leq_{\mathbb{P} \uparrow B} p_{0}$.

Proof. By recursion-induction on $\alpha$, simultaneously for all templates $(L, \mathcal{I})$,

- we prove that $\mathbb{P} \mid B$ is indeed a p.o. (i.e., transitivity holds) for all $B \in \mathcal{I}$ with $\operatorname{Dp}(B)=\alpha$;

- we prove $\mathbb{P}\lceil A \subset \mathbb{P}\lceil B$ for all $B \in \mathcal{I}$ with $\operatorname{Dp}(B)=\alpha$ and all closed $A \subset B$;

- we construct $p_{0}=p_{0}(p, A, B)$ satisfying (i) and (ii) for all $B \in \mathcal{I}$ with $\operatorname{Dp}(B)=\alpha$, all closed $A \subset B$ and all $p \in \mathbb{P}\lceil B$;

- we prove that for all $B, D \in \mathcal{I}, B, C \subseteq D, C$ closed, with $\operatorname{Dp}(D)=\alpha$ and all $p \in \mathbb{P} \mid B$, letting $A=C \cap B$ and $p_{0}=p_{0}(p, A, B)$ (which has been constructed either at stage $\alpha$ or at an earlier stage), there is $q \in \mathbb{P} \mid D$ as required.

Notice that if $\operatorname{Dp}(B)=\alpha, D=B$ and $C=A$, then the latter indeed shows $\mathbb{P}\lceil A<\circ \mathbb{P} \uparrow B$.

The case $\alpha=0$ is trivial. So assume $\alpha>0$ and $\operatorname{Dp}(B)=\alpha$. We first check transitivity of $\mathbb{P}\left\lceil B\right.$. Assume that $r \leq_{\mathbb{P} \mid B} q \leq_{\mathbb{P} \mid B} p$. Then clearly $\operatorname{dom}(r) \supseteq \operatorname{dom}(p)$, $n^{r} \geq n^{p}, p(z) \subseteq r(z)$ for all $z \in \operatorname{dom}(p) \cap L_{\text {mad }}$, and $\mid\left\{z \in \operatorname{dom}(p) \cap L_{\text {mad }} ; r(z)(i)=\right.$ $1\} \mid \leq 1$ for all $i \in n^{r} \backslash n^{p}$. Let $z=\max \left(\operatorname{dom}(r) \cap L_{\mathrm{Hech}}\right), y=\max (\operatorname{dom}(q) \cap$ $\left.L_{\text {Hech }}\right)$ and $x=\max \left(\operatorname{dom}(p) \cap L_{\text {Hech }}\right)$. Then there are $A_{0}, A_{1} \in \mathcal{I} \cap \mathcal{P}\left(B \cap L_{z}\right)$ 
such that $p \uparrow\left(B \cap L_{z}\right), q \uparrow\left(B \cap L_{z}\right) \in \mathbb{P}\left\lceil A_{0}, q \uparrow\left(B \cap L_{z}\right) \leq \mathbb{P} \uparrow A_{0} \quad p \uparrow\left(B \cap L_{z}\right)\right.$, and $q \uparrow\left(B \cap L_{z}\right), r \uparrow\left(B \cap L_{z}\right) \in \mathbb{P}\left\lceil A_{1}, r \uparrow\left(B \cap L_{z}\right) \leq_{\mathbb{P} \uparrow A_{1}} q \uparrow\left(B \cap L_{z}\right)\right.$. Furthermore, $\dot{f}_{z}^{r}$ is a $\mathbb{P} \nmid A_{1}$-name and, in case $y=z, \dot{f}_{y}^{q}$ is both a $\mathbb{P}\left\lceil A_{1}\right.$-name and a $\mathbb{P} \nmid A_{0}$-name as well as, in case $x=y=z, \dot{f}_{x}^{p}$ is a $\mathbb{P} \mid A_{0}$-name. Let $A=A_{0} \cup A_{1}$. Then $A \in \mathcal{I} \cap \mathcal{P}\left(B \cap L_{z}\right)$ so that $\operatorname{Dp}(A)<\operatorname{Dp}(B)$, and we know by the induction hypothesis that $\mathbb{P}\left\lceil A_{i}<0 \mathbb{P}\left\lceil A\right.\right.$ for $i=0,1$. Therefore, $p \nmid\left(B \cap L_{z}\right), q \uparrow\left(B \cap L_{z}\right), r \uparrow\left(B \cap L_{z}\right) \in \mathbb{P}\lceil A$ and $r \uparrow\left(B \cap L_{z}\right) \leq_{\mathbb{P} \uparrow A} q \uparrow\left(B \cap L_{z}\right) \leq_{\mathbb{P} \uparrow A} p \uparrow\left(B \cap L_{z}\right)$. Moreover, $\dot{f}_{z}^{r}$ is a $\mathbb{P}\lceil A$-name and, in case $y=z, \dot{f}_{y}^{q}$ is a $\mathbb{P}\left\lceil A\right.$-name and $r \uparrow\left(B \cap L_{z}\right) \vdash_{\mathbb{P} \uparrow A} \dot{f}_{y}^{q} \leq \dot{f}_{z}^{r}$ as well as, in case $x=y=z, \dot{f}_{x}^{p}$ is a $\mathbb{P}\left\lceil A\right.$-name and $q \uparrow\left(B \cap L_{z}\right) \Vdash_{\mathbb{P} \uparrow A} \dot{f}_{x}^{p} \leq \dot{f}_{y}^{q}$. Taken together, this shows that $r \leq_{\mathbb{P} \mid B} p$ as required.

Now let $A \subset B \in \mathcal{I}, A$ closed, be given. Assume $r \in \mathbb{P} \mid A$. Let $x=\max (\operatorname{dom}(r) \cap$ $\left.L_{\mathrm{Hech}}\right)$. By definition of the iteration there is $\bar{A} \in\left(\mathcal{I}\lceil A) \cap \mathcal{P}\left(L_{x}\right)\right.$ such that $r \uparrow(A \cap$ $\left.L_{x}\right) \in \mathbb{P}\left\lceil\bar{A}\right.$ and $\dot{f}_{x}^{r}$ is a $\mathbb{P}\lceil\bar{A}$-name. There is $\bar{B} \in \mathcal{I} \mid B \subseteq \mathcal{I}$ such that $\bar{A}=A \cap \bar{B}$. Clearly $x \notin \bar{B}$. By clause $\left(4^{\prime}\right)$ in the definition of a template, we may therefore assume without loss of generality that $\bar{B} \subseteq L_{x}$. Thus $\bar{B} \subset B$ and $\operatorname{Dp}(\bar{B})<$ $\operatorname{Dp}(B)=\alpha$. By induction hypothesis, $\mathbb{P}\lceil\bar{A} \subset \mathbb{P}\lceil\bar{B}$ and $\mathbb{P}\lceil\bar{A}<0 \mathbb{P}\lceil\bar{B}$. Therefore, $\dot{f}_{x}^{r}$ is a $\mathbb{P}\lceil\bar{B}$-name as well. $r \in \mathbb{P}\lceil B$ follows immediately. Hence $\mathbb{P}\lceil A \subset \mathbb{P}\lceil B$ as required.

Next assume also $p \in \mathbb{P}\left\lceil B\right.$ is given. We construct $p_{0}=p_{0}(p, A, B)$. Put $x=$ $\max \left(\operatorname{dom}(p) \cap L_{\mathrm{Hech}}\right)$. By definition of the iteration there is $\bar{B} \in \mathcal{I} \cap \mathcal{P}\left(B \cap L_{x}\right)$ such that $\bar{p}=p\left\lceil\left(B \cap L_{x}\right) \in \mathbb{P}\left\lceil\bar{B}\right.\right.$ and $\dot{f}_{x}^{p}$ is a $\mathbb{P}\lceil\bar{B}$-name. Put $\bar{A}=A \cap \bar{B}$. Note that $\bar{A} \in \mathcal{I}\left\lceil A\right.$. By induction hypothesis, $\bar{p}$ has a reduction $\bar{p}_{0}=p_{0}(\bar{p}, \bar{A}, \bar{B}) \in \mathbb{P}\lceil\bar{A}$ satisfying the barred version of the clauses of the main lemma. The definition of $p_{0}$ splits into two cases.

Case 1. $x \notin A$. Then $\operatorname{dom}\left(p_{0}\right)=\operatorname{dom}(p) \cap A, p_{0} \uparrow\left(A \cap L_{x}\right)=\bar{p}_{0}$ and $p_{0}(z)=p(z)$ for $z \in(\operatorname{dom}(p) \cap A) \backslash L_{x}$. (Note that such $z$ must belong to $L_{\text {mad. }}$.)

Case 2. $x \in A$. Then let $\operatorname{dom}\left(p_{0}\right)=\operatorname{dom}(p) \cap A, p_{0} \uparrow\left(A \cap L_{x}\right)=\bar{p}_{0}$ and $p_{0}(z)=p(z)$ for $z \in\left(\operatorname{dom}(p) \cap A \cap L_{\text {mad }}\right) \backslash L_{x}$. We know by induction hypothesis that $\mathbb{P}\lceil\bar{A}$ $<\circ \mathbb{P}\left\lceil\bar{B}\right.$. Therefore, there exists a canonical projection $\dot{f}_{x}^{p_{0}}$ to $\mathbb{P}\lceil\bar{A}$ of the $\mathbb{P}\lceil\bar{B}-$ name $\dot{f}_{x}^{p}$. Accordingly we let $p_{0}(x)=\left(s_{x}^{p}, \dot{f}_{x}^{p_{0}}\right)$.

More explicitly, we do the following. For simplicity work with the $\mathrm{cBa}$ 's $\mathbb{B}_{\bar{A}}=$ r.o. $\left(\mathbb{P}\lceil\bar{A})\right.$ and $\mathbb{B}_{\bar{B}}=$ r.o. $(\mathbb{P}\lceil\bar{B})$ associated with $\bar{A}$ and $\bar{B}$. We know by the induction hypothesis that $\mathbb{B}_{\bar{A}}<0 \mathbb{B}_{\bar{B}}$. Note that $\bar{p} \leq \llbracket s_{x}^{p} \subseteq \dot{f}_{x}^{p} \rrbracket$. In $\mathbb{B}_{\bar{B}}$, for all $s \in \omega^{<\omega}$ with $s_{x}^{p} \subseteq s$, we let $b_{s}=\llbracket s \subseteq \dot{f}_{x}^{p} \rrbracket \cap \bar{p}$. So $b_{s_{x}^{p}}=\bar{p}$ and, for $n>\left|s_{x}^{p}\right|$, the $b_{s}$, $|s|=n$, are a maximal antichain below $\bar{p}$. Let $a_{s}^{*}$ be the product (intersection) of $\bar{p}_{0}$ and the projection of $b_{s}$ to $\mathbb{B}_{\bar{A}}$ (recall the projection of $b_{s}$ to $\mathbb{B}_{\bar{A}}$ is the unique condition $a$ such that $a \geq b_{s}$ and any extension of $a$ in $\mathbb{B}_{\bar{A}}$ is compatible with $b_{s}$ ). In particular, $a_{s_{x}^{p}}^{*}=\bar{p}_{0}$ and $\sum\left\{a_{s}^{*} ;|s|=n\right\}=\bar{p}_{0}$ for $n>\left|s_{x}^{p}\right|$. Define $a_{s}$ by recursion on $n=|s|$ as follows. $a_{s_{x}^{p}}=a_{s_{x}^{p}}^{*}=\bar{p}_{0}$ and, for $n>\left|s_{x}^{p}\right|$, set $a_{s}=a_{s \uparrow(n-1)} \cdot\left(a_{s}^{*} \mid\right.$ $\left.\sum_{j<s(n-1)} a_{s \uparrow(n-1)^{\wedge}\langle j\rangle}^{*}\right)$ (which is equal to $a_{s \uparrow(n-1)} \cdot\left(a_{s}^{*} \backslash \sum_{j<s(n-1)} a_{s \uparrow(n-1)^{\wedge}\langle j\rangle}\right)$ ). Then one can show by induction on $n>\left|s_{x}^{p}\right|$ that the $a_{s},|s|=n$, are a maximal antichain below $\bar{p}_{0}$. Therefore they canonically define a $\mathbb{P} \uparrow \bar{A}$-name $\dot{f}_{x}^{p_{0}}$ (that is, $\left.a_{s}=\llbracket s \subseteq \dot{f}_{x}^{p_{0}} \rrbracket\right)$ such that $\bar{p}_{0} \Vdash_{\mathbb{P} \uparrow \bar{A}} \dot{f}_{x}^{p_{0}} \in \omega^{\omega}$.

The main property of this name is that for all $s, a_{s}^{\prime}=\sum\left\{a_{s^{\prime}} ; s^{\prime} \leq s\right.$ everywhere, $\left.s_{x}^{p} \subseteq s,\left|s^{\prime}\right|=|s|\right\}$ is a reduction (not necessarily the projection) of $b_{s}^{\prime}=\sum\left\{b_{s^{\prime}} ; s^{\prime} \leq\right.$ $s$ everywhere, $\left.s_{x}^{p} \subseteq s,\left|s^{\prime}\right|=|s|\right\}$. (This is so because $\left(a_{s}^{*}\right)^{\prime}=\sum\left\{a_{s^{\prime}}^{*} ; s^{\prime} \leq s\right.$ 
everywhere, $\left.s_{x}^{p} \subseteq s,\left|s^{\prime}\right|=|s|\right\}$ is the product of $\bar{p}_{0}$ and the projection of $b_{s}^{\prime}$ and, by the definition of $a_{s}, a_{s}^{\prime} \leq\left(a_{s}^{*}\right)^{\prime}$ is trivial.)

This completes the definition of $p_{0}$. Clauses (i) and (ii) are trivially satisfied in each of the two cases.

Now assume $B, D \in \mathcal{I}, B, C \subseteq D, C$ closed, are such that $\operatorname{Dp}(D)=\alpha, A=C \cap B$, $p \in \mathbb{P}\left\lceil B\right.$ and $p_{0}=p_{0}(p, A, B)$. Let $q_{0} \leq_{\mathbb{P} \nmid C} p_{0}, q_{0} \in \mathbb{P}\lceil C$. We need to construct $q$. $x=\max \left(\operatorname{dom}(p) \cap L_{\mathrm{Hech}}\right), \bar{B}, \bar{A}, \bar{p}$ and $\bar{p}_{0}$ are as in the previous construction.

Case 1. $x \notin A$. So $x \notin C$. Let $y=\max \left\{z<x ; z \in \operatorname{dom}\left(q_{0}\right) \cap L_{\mathrm{Hech}}\right\}$. We can find $\bar{E} \in \mathcal{I}\left\lceil C \cap \mathcal{P}\left(L_{y}\right)\right.$ such that $q_{0} \uparrow\left(C \cap L_{y}\right) \in \mathbb{P}\left\lceil\bar{E}\right.$ and $\dot{f}_{y}^{q_{0}}$ is a $\mathbb{P}\lceil\bar{E}$-name. There is $\bar{F} \in \mathcal{I} \backslash D \subseteq \mathcal{I}$ such that $\bar{E}=\bar{F} \cap C$. Since $y \notin \bar{E}$ and $y \in C, y \notin \bar{F}$ follows. By clause $\left(4^{\prime}\right)$ in the definition of a template, without loss of generality, $\bar{F} \subseteq L_{y}$. By clause $\left(3^{\prime}\right)$ in the definition of a template find $\bar{G} \in(\mathcal{I} \backslash D) \cap \mathcal{P}\left(L_{x}\right) \subseteq \mathcal{I}$ containing $y$. Let $\bar{D}=\bar{G} \cup \bar{F} \cup \bar{B} \subseteq L_{x}$ and $\bar{C}=(\bar{G} \cap C) \cup \bar{E} \cup \bar{A} \subseteq L_{x}$. By clause (2) in the definition of a template, $\bar{D} \in \mathcal{I}\lceil D \subseteq \mathcal{I}$ and $\bar{C} \in \mathcal{I}\lceil C$. Since $\operatorname{Dp}_{\mathcal{I} \mid C}(\bar{E}) \leq \operatorname{Dp}_{\mathcal{I}\lceil C}(\bar{C}) \leq \operatorname{Dp}_{\mathcal{I}}(\bar{D})<\alpha, \bar{q}_{0}=q_{0} \uparrow\left(C \cap L_{x}\right) \in \mathbb{P}\lceil\bar{C}$ by the induction hypothesis. $\bar{q}_{0} \leq_{\mathbb{P} \uparrow \bar{C}} \bar{p}_{0}$ and $\bar{C} \cap \bar{B}=\bar{A}$ are immediate. By the inductive assumption for the barred version, there is $\bar{q} \in \mathbb{P}\left\lceil\bar{D}\right.$ extending both $\bar{q}_{0}$ and $\bar{p}$.

We define $q$ such that

- $\operatorname{dom}(q)=\operatorname{dom}(\bar{q}) \cup \operatorname{dom}(p) \cup \operatorname{dom}\left(q_{0}\right), n^{q}=n^{\bar{q}}$,

- $q \uparrow\left(D \cap L_{x}\right)=\bar{q}$,

- $q(y)=q_{0}(y)$ for all $y \in\left(\operatorname{dom}\left(q_{0}\right) \backslash L_{x}\right) \cap L_{\mathrm{Hech}}$,

- $q(x)=p(x)$,

- $q_{0}(z) \subseteq q(z), q(z)(i)=0$, for $z \in\left(\operatorname{dom}\left(q_{0}\right) \backslash L_{x}\right) \cap L_{\operatorname{mad}}$ and $i \in n^{q} \backslash n^{q_{0}}$,

- $p(z) \subseteq q(z), q(z)(i)=0$, for $z \in\left(\operatorname{dom}(p) \backslash\left(L_{x} \cup \operatorname{dom}\left(q_{0}\right)\right)\right) \cap L_{\text {mad }}$ and $i \in n^{q} \backslash n^{p}$.

It is straightforward to check that $q \in \mathbb{P}\left\lceil D\right.$ and $q \leq_{\mathbb{P} \nmid D} q_{0}$. So let us argue that $q \leq \mathbb{P} \mid D p$ as well. Clearly $n^{q} \geq n^{p}$. We need to show that $p(z) \subseteq q(z)$ for all $z \in \operatorname{dom}(p) \cap L_{\text {mad }}$. This is obvious for $z<x$ because $\bar{q} \leq_{\mathbb{P} \uparrow \bar{D}} \bar{p}$. It is immediate by definition for $z>x$ belonging to $\operatorname{dom}(p) \backslash \operatorname{dom}\left(q_{0}\right)$. So assume $z>x, z \in$ $\operatorname{dom}(p) \cap \operatorname{dom}\left(q_{0}\right)$. Then $p(z)=p_{0}(z) \subseteq q_{0}(z) \subseteq q(z)$, as required. Next fix $i \in n^{q} \backslash n^{p}$. We need to check that there is at most one $z \in \operatorname{dom}(p) \cap L_{\text {mad }}$ with $q(z)(i)=1$. By way of contradiction assume this is true for two distinct $z_{0}<z_{1}$. By construction we must have $i \in n^{q_{0}}, x<z_{1}$ and $z_{1} \in \operatorname{dom}\left(q_{0}\right) \cap \operatorname{dom}(p)$. Hence $z_{1} \in A$. Therefore $z_{0}$ must belong to $A$ as well because $A$ is closed. Thus both $z_{0}$ and $z_{1}$ belong to $\operatorname{dom}\left(p_{0}\right)$. This means that $q\left(z_{j}\right)(i)=q_{0}\left(z_{j}\right)(i)=1$ for $j=0,1$, which contradicts $q_{0} \leq \mathbb{P} \uparrow C p_{0}$, and we are done.

Case 2. $x \in A$. So $x \in C$. Find $\bar{C} \in \mathcal{I}\left\lceil C \cap \mathcal{P}\left(L_{x}\right)\right.$ such that $\bar{q}_{0}=q_{0} \uparrow\left(C \cap L_{x}\right) \in \mathbb{P}\lceil\bar{C}$ and $\dot{f}_{x}^{q_{0}}$ is a $\mathbb{P}\lceil\bar{C}$-name. Without loss of generality, $\bar{A} \subseteq \bar{C}$. $\bar{C} \cap \bar{B}=\bar{A}$ is immediate. There is $\bar{D} \in \mathcal{I} \mid D \subseteq \mathcal{I}$ such that $\bar{C}=\bar{D} \cap C$. Since $x \notin \bar{C}$, we get $x \notin \bar{D}$. By $\left(4^{\prime}\right)$, without loss of generality, $\bar{D} \subseteq L_{x}$. We may also assume $\bar{B} \subseteq \bar{D}$. Since $\mathrm{Dp}_{\mathcal{I}}(\bar{D})<\mathrm{Dp}_{\mathcal{I}}(D)=\alpha$, we can freely use the induction hypothesis when dealing with $\bar{A}, \bar{B}, \bar{C}$, and $\bar{D}$. In particular, $\bar{q}_{0} \leq_{\mathbb{P} \uparrow \bar{C}} \bar{p}_{0}$.

Now note that we have $s_{x}^{p}=s_{x}^{p_{0}} \subseteq s_{x}^{q_{0}}$ and $\bar{q}_{0} \Vdash_{\mathbb{P} \mid \bar{C}} s_{x}^{q_{0}} \subseteq \dot{f}_{x}^{q_{0}}$. Let $m=\left|s_{x}^{q_{0}}\right|$. Since also $\bar{q}_{0} \Vdash_{\mathbb{P} \uparrow \bar{C}}$ " $\dot{f}_{x}^{q_{0}} \geq \dot{f}_{x}^{p_{0}}$ (everywhere)", we see that $\bar{q}_{0} \Vdash_{\mathbb{P} \uparrow \bar{C}} \dot{f}_{x}^{p_{0}}\left\lceil m \leq s_{x}^{q_{0}}\right.$. Hence we get $a:=a_{s_{x}^{q_{0}}}^{\prime}=\llbracket \dot{f}_{x}^{p_{0}}\left\lceil m \leq s_{x}^{q_{0}} \rrbracket \geq_{\mathbb{P} \uparrow \bar{A}} \bar{p}_{0}^{*}\right.$ where we let $\bar{p}_{0}^{*}=p_{0}\left(\bar{q}_{0}, \bar{A}, \bar{C}\right)$ (the canonical reduction of $\bar{q}_{0}$ to $\mathbb{P}\left\lceil\bar{A}\right.$; note here that $\operatorname{Dp}_{\mathcal{I} \mid \bar{C}}(\bar{C}) \leq \operatorname{Dp}_{\mathcal{I}}(\bar{D})<\alpha$, so 
that $\bar{p}_{0}^{*}$ indeed has been defined already). However, by construction, $a \leq_{\mathbb{P} \mid \bar{A}} \bar{p}_{0}$ is nothing but a reduction of $b:=b_{s_{x}^{q_{0}}}^{\prime}=\llbracket \dot{f}_{x}^{p}\left\lceil m \leq s_{x}^{q_{0}} \rrbracket \cap \bar{p} \in \mathbb{B}_{\bar{B}}\right.$ to $\mathbb{B}_{\bar{A}}$. So there is $\bar{p}^{+} \in \mathbb{P}\left\lceil\bar{B}\right.$ such that $\bar{p}^{+} \leq_{\mathbb{P}\lceil\bar{B}} \bar{p}_{0}^{*}$ and $\bar{p}^{+} \leq_{\mathbb{P}\lceil\bar{B}} b$ (so that, in particular, $\bar{p}^{+} \leq_{\mathbb{P}\lceil\bar{B}} \bar{p}$ and $\bar{p}^{+} \Vdash_{\mathbb{P}\lceil\bar{B}} \dot{f}_{x}^{p}\left\lceil m \leq s_{x}^{q_{0}}\right)$. Let $\bar{p}_{0}^{+}=p_{0}\left(\bar{p}^{+}, \bar{A}, \bar{B}\right)$ be the canonical reduction of $\bar{p}^{+}$to $\mathbb{P}\left\lceil\bar{A}\right.$. Then $\bar{p}_{0}^{+} \leq_{\mathbb{P} \uparrow \bar{A}} \bar{p}_{0}^{*}$. Therefore $\bar{p}_{0}^{+}$and $\bar{q}_{0}$ have a common extension $\bar{q}_{0}^{+}$in $\mathbb{P}\left\lceil\bar{C}\right.$. By inductive assumption for the barred +- -version, there is $\bar{q}^{+} \in \mathbb{P}\lceil\bar{D}$ extending both $\bar{p}^{+}$and $\bar{q}_{0}^{+}$.

We define $q$ such that

- $\operatorname{dom}(q)=\operatorname{dom}\left(\bar{q}^{+}\right) \cup \operatorname{dom}(p) \cup \operatorname{dom}\left(q_{0}\right), n^{q}=n^{\bar{q}^{+}}$,

- $q \uparrow\left(D \cap L_{x}\right)=\bar{q}^{+}$,

- $q(y)=q_{0}(y)$ for all $y \in \operatorname{dom}\left(q_{0}\right) \cap L_{\text {Hech }}$ with $y>x$,

- $s_{x}^{q}=s_{x}^{q_{0}}$ and $\dot{f}_{x}^{q}$ is a $\mathbb{P}\left\lceil\bar{D}\right.$-name such that $\bar{q}^{+} \Vdash_{\mathbb{P} \uparrow \bar{D}} \dot{f}_{x}^{q}=\max \left\{\dot{f}_{x}^{q_{0}}, \dot{f}_{x}^{p}\right\}$,

- $q_{0}(z) \subseteq q(z), q(z)(i)=0$, for $z \in\left(\operatorname{dom}\left(q_{0}\right) \backslash L_{x}\right) \cap L_{\operatorname{mad}}$ and $i \in n^{q} \backslash n^{q_{0}}$,

- $p(z) \subseteq q(z), q(z)(i)=0$, for $z \in\left(\operatorname{dom}(p) \backslash\left(L_{x} \cup \operatorname{dom}\left(q_{0}\right)\right)\right) \cap L_{\mathrm{mad}}$ and $i \in n^{q} \backslash n^{p}$.

To see that $q \in \mathbb{P}\left\lceil D\right.$, note that $\bar{q}^{+} \Vdash_{\mathbb{P}\lceil\bar{D}} s_{x}^{q_{0}} \subseteq \dot{f}_{x}^{q}$ by construction. It is then straightforward to check that $q \leq_{\mathbb{P} \mid D} q_{0}, p$. In fact, for $q \leq_{\mathbb{P} \mid D} p$ we argue as in Case 1 above.

Note that, as an immediate consequence of Main Lemma 1.1, we get that for arbitrary closed $A \subseteq B \subseteq L, \mathbb{P} \uparrow(A, \mathcal{I}\lceil A)$ completely embeds into $\mathbb{P} \uparrow(B, \mathcal{I}\lceil B)$.

Lemma 1.2 (Chain condition). Let $A \in \mathcal{I}$. Any uncountable $K \subseteq \mathbb{P}\lceil A$ has an uncountable centered subset.

Proof. By a standard $\Delta$-system argument, it suffices to show that if $p, q \in \mathbb{P}\lceil A$, $n^{p}=n^{q}, s_{x}^{p}=s_{x}^{q}$ for all $x \in \operatorname{dom}(p) \cap \operatorname{dom}(q) \cap L_{\mathrm{Hech}}$, and $p(x)=q(x)$ for all $x \in \operatorname{dom}(p) \cap \operatorname{dom}(q) \cap L_{\text {mad }}$, then there is a common extension $r$ with $\operatorname{dom}(r)=$ $\operatorname{dom}(p) \cup \operatorname{dom}(q), n^{r}=n^{p}=n^{q}$,

$$
s_{x}^{r}= \begin{cases}s_{x}^{p} & \text { if } x \in \operatorname{dom}(p) \cap L_{\mathrm{Hech}}, \\ s_{x}^{q} & \text { if } x \in \operatorname{dom}(q) \cap L_{\mathrm{Hech}}\end{cases}
$$

and

$$
r(x)=\left\{\begin{array}{cl}
p(x) & \text { if } x \in \operatorname{dom}(p) \cap L_{\mathrm{mad}} \\
q(x) & \text { if } x \in \operatorname{dom}(q) \cap L_{\mathrm{mad}}
\end{array}\right.
$$

We do this by induction on $\operatorname{Dp}(A)$.

The case $\operatorname{Dp}(A)=0$ is trivial. So assume $\operatorname{Dp}(A)>0$. First assume $x=$ $\max \left(\operatorname{dom}(p) \cap L_{\mathrm{Hech}}\right)<y=\max \left(\operatorname{dom}(q) \cap L_{\mathrm{Hech}}\right)$. Then there is $B \in \mathcal{I} \cap \mathcal{P}\left(A \cap L_{y}\right)$ such that $p\left\lceil L_{y}, q\left\lceil L_{y} \in \mathbb{P}\left\lceil B\right.\right.\right.$, and $\dot{f}_{y}^{q}$ is a $\mathbb{P}\lceil B$-name. By the induction hypothesis, we get the required $r\left\lceil L_{y} \leq_{\mathbb{P}\lceil B} p\left\lceil L_{y}, q\left\lceil L_{y}\right.\right.\right.$. Let $r(y)=q(y)$ and let $r(z)=p(z)$ for $z \in \operatorname{dom}(p) \cap L_{\mathrm{mad}}, z>y$, and $r(z)=q(z)$ for $z \in(\operatorname{dom}(q) \backslash \operatorname{dom}(p)) \cap L_{\text {mad }}, z>y$.

Next assume $x=\max \left(\operatorname{dom}(p) \cap L_{\mathrm{Hech}}\right)=\max \left(\operatorname{dom}(q) \cap L_{\mathrm{Hech}}\right)$. Again there is $B \in \mathcal{I} \cap \mathcal{P}\left(A \cap L_{x}\right)$ such that $p\left\lceil L_{x}, q\left\lceil L_{x} \in \mathbb{P}\left\lceil B\right.\right.\right.$, and $\dot{f}_{x}^{p}, \dot{f}_{x}^{q}$ are $\mathbb{P}\lceil B$-names. Again we get $r\left\lceil L_{x}\right.$. Let $s_{x}^{r}=s_{x}^{p}=s_{x}^{q}$ and $\dot{f}_{x}^{r}$ be such that $r\left\lceil L_{x} \Vdash_{\mathbb{P} \uparrow B} \dot{f}_{x}^{r}=\max \left\{\dot{f}_{x}^{p}, \dot{f}_{x}^{q}\right\}\right.$. Also let $r(z)=p(z)$ for $z \in \operatorname{dom}(p) \cap L_{\mathrm{mad}}, z>y$, and $r(z)=q(z)$ for $z \in$ $(\operatorname{dom}(q) \backslash \operatorname{dom}(p)) \cap L_{\mathrm{mad}}, z>y$.

Lemma 1.3 (Embedding Hechler forcing). Let $x \in L_{\mathrm{Hech}}$ and $A \in \mathcal{I} \cap \mathcal{P}\left(L_{x}\right)$. Then the two-step iteration $\mathbb{P}\left\lceil A \star \dot{\mathbb{D}}_{x}\right.$ that canonically adds a Hechler-generic in coordinate $x$ over the generic extension via $\mathbb{P}\lceil A$ completely embeds into $\mathbb{P}\lceil L$. 
Proof. Let $B=\operatorname{cl}(A \cup\{x\}) . \mathbb{P}\lceil B$ embeds into $\mathbb{P}\lceil L$ by Main Lemma 1.1 So it suffices to show $\mathbb{P}\left\lceil A \star \dot{\mathbb{D}}_{x}<0 \mathbb{P}\lceil B\right.$. This does not follow from (the statement of) Lemma 1.1 because $A \cup\{x\}$ need not be closed, but it is relatively straightforward from the proof of 1.1 .

More explicitly, given $p \in \mathbb{P}\left\lceil B\right.$, there is $\bar{B} \in \mathcal{I} \mid B \cap \mathcal{P}\left(L_{x}\right)$ such that $\bar{p}=p\left\lceil L_{x} \in\right.$ $\mathbb{P}\left\lceil\bar{B}\right.$ and $\dot{f}_{x}^{p}$ is a $\mathbb{P}\lceil\bar{B}$-name. Without loss of generality, $A \subseteq \bar{B}$. By 1.1, $\mathbb{P}\lceil A<$ $\circ \mathbb{P}\left\lceil\bar{B}\right.$. Therefore, $\bar{p}$ has a canonical reduction $\bar{p}_{0} \in \mathbb{P}\lceil A$. As in Case 2 of the proof of 1.1 there is a canonical projection $\dot{f}_{x}^{p_{0}}$ to $\mathbb{P}\left\lceil A\right.$ of $\dot{f}_{x}^{p}$. Define $p_{0} \in \mathbb{P}\left\lceil A \star \dot{\mathbb{D}}_{x}\right.$ by $p_{0}\left\lceil A=\bar{p}_{0}\right.$ and $p_{0}(x)=\left(s_{x}^{p}, \dot{f}_{x}^{p_{0}}\right)$. As in Case 2 of the proof of 1.1, argue that any $q_{0} \in \mathbb{P}\left\lceil A \star \dot{\mathbb{D}}_{x}\right.$ extending $p_{0}$ is compatible with $p$.

This may badly fail in case $A \notin \mathcal{I}$ because then $\mathbb{P} \mid A \star \dot{\mathbb{D}}_{x}$ need not embed into $\mathbb{P} \uparrow B$.

Lemma 1.4 (Names for reals). Assume $p \in \mathbb{P}\lceil L$ and $\dot{f}$ is a $\mathbb{P}\lceil L$-name for a real. Then there is $A \subseteq L$ countable such that, letting $B=\operatorname{cl}(A), p \in \mathbb{P} \mid B$, and $\dot{f}$ is a $\mathbb{P} \mid B$-name.

Proof. The proof proceeds by simultaneous induction on $\operatorname{Dp}(L)$. Without loss of generality, $\operatorname{Dp}(L)>0$.

Assume first $p \in \mathbb{P} \mid L$. Let $x=\max \left(\operatorname{dom}(p) \cap L_{\text {Hech }}\right)$. There is $C \in \mathcal{I} \cap \mathcal{P}\left(L_{x}\right)$ such that $p\left\lceil L_{x} \in \mathbb{P}\left\lceil C\right.\right.$ and $\dot{f}_{x}^{p}$ is a $\mathbb{P}\lceil C$-name. By induction hypothesis, there is $A_{0} \subseteq C$ countable such that $p \nmid L_{x} \in \mathbb{P}\left\lceil\mathrm{cl}\left(A_{0}\right)\right.$ and $\dot{f}_{x}^{p}$ is a $\mathbb{P}\left\lceil\mathrm{cl}\left(A_{0}\right)\right.$-name. Then $p \in \mathbb{P} \mid B$ where $B=\operatorname{cl}(A), A=A_{0} \cup \operatorname{dom}(p)$.

Assume now that $\dot{f}$ is a $\mathbb{P}\left\lceil L\right.$-name. By ccc-ness (Lemma 1.2), there are $\left\{p_{n, i}\right.$; $i, n \in \omega\} \subseteq \mathbb{P}\left\lceil L\right.$ and $\left\{k_{n, i} \in \omega ; i, n \in \omega\right\}$ such that

- $p_{n, i} \Vdash_{\mathbb{P}\lceil L} \dot{f}(n)=k_{n, i}$,

- $\left\{p_{n, i} ; i \in \omega\right\}$ is a maximal antichain in $\mathbb{P}\lceil L$ for all $n \in \omega$.

By the previous paragraph, we can find countable sets $A_{n, i}$ such that $p_{n, i} \in$ $\mathbb{P}\left\lceil\mathrm{cl}\left(A_{n, i}\right)\right.$. Put $A=\bigcup_{i, n} A_{n, i}, B=\operatorname{cl}(A)$. Since $\mathbb{P}\lceil B<0 \mathbb{P}\lceil L$ (Lemma 1.1), we can construe $\dot{f}$ as a $\mathbb{P}\lceil B$-name.

Assume $(L, \mathcal{I})$ and $(L, \mathcal{J})$ are templates and $\mathcal{I} \subseteq \mathcal{J}$. We say that $\mathcal{I}$ is cofinal in $\mathcal{J}$ if for all $x \in L_{\mathrm{Hech}}$ and all $A \in \mathcal{J} \cap \mathcal{P}\left(L_{x}\right)$ there is $B \in \mathcal{I} \cap \mathcal{P}\left(L_{x}\right)$ containing $A$. The following is, in a sense, a triviality.

Lemma 1.5 (Cofinal subtemplates). If $\mathcal{I}$ is cofinal in $\mathcal{J}$, then $\mathbb{P} \uparrow(L, \mathcal{I})$ is forcing equivalent to $\mathbb{P} \uparrow(L, \mathcal{J})$.

Proof. By induction on $\operatorname{Dp}(L)$ (in the sense of $\mathcal{I}$ ), we argue that conditions in $\mathbb{P} \uparrow(L, \mathcal{I})$ and conditions in $\mathbb{P} \uparrow(L, \mathcal{J})$ can be canonically identified so as to yield forcing equivalence. Without loss of generality, $\mathrm{Dp}(L)>0$.

Let $p \in \mathbb{P} \uparrow(L, \mathcal{J})$. Put $x=\max \left(\operatorname{dom}(p) \cap L_{\text {Hech }}\right)$. There is $A \in \mathcal{J} \cap \mathcal{P}\left(L_{x}\right)$ such that $\bar{p}=p\left\lceil L_{x} \in \mathbb{P} \uparrow(A, \mathcal{J})\right.$ and $\dot{f}_{x}^{p}$ is a $\mathbb{P} \uparrow(A, \mathcal{J})$-name. Since $\mathcal{I}$ is cofinal in $\mathcal{J}$, there is $B \in \mathcal{I} \cap \mathcal{P}\left(L_{x}\right)$ such that $A \subseteq B$. By Main Lemma 1.1 we know that $\mathbb{P} \uparrow(A, \mathcal{J})<\circ \mathbb{P} \uparrow(B, \mathcal{J})$ and, by the induction hypothesis, $\mathbb{P} \uparrow(B, \mathcal{J})$ and $\mathbb{P} \uparrow(B, \mathcal{I})$ are forcing equivalent. Therefore, we may construe $\bar{p}$ as a condition in $\mathbb{P} \uparrow(B, \mathcal{I})$ and $\dot{f}_{x}^{p}$ as a $\mathbb{P} \uparrow(B, \mathcal{I})$-name. Thus $p \in \mathbb{P} \uparrow(L, \mathcal{I})$. It is straightforward to verify that this identification induces forcing equivalence. 
Proposition 1.6 (Adjoining a scale). Assume $\mu$ is regular uncountable, $\mu \subseteq L_{\mathrm{Hech}}$ is cofinal in $L$, and $L_{\alpha} \in \mathcal{I}$ for all $\alpha<\mu$. Then $\mathbb{P} \mid L$ forces $\mathfrak{b}=\mathfrak{d}=\mu$ (i.e., there is a $\mu$-scale).

Proof. For each $\alpha<\mu$, let $\dot{f}_{\alpha}$ be the name for the Hechler-generic adjoined in coordinate $\alpha$ of the iteration (see Lemma 1.3). By construction, the $\dot{f}_{\alpha}$ are forced to be well-ordered by $\leq^{*}$. Let $\dot{g}$ be a $\mathbb{P} \mid L$-name for a real. By Lemma 1.4 there is $A \subseteq L$ countable such that $\dot{g}$ is a $\mathbb{P} \mid \mathrm{cl}(A)$-name. Since $\mu$ is regular uncountable and cofinal in $L$, there is $\alpha<\mu$ such that $\operatorname{cl}(A) \subseteq L_{\alpha}$. Since $L_{\alpha} \in \mathcal{I}, \dot{f}_{\alpha}$ is forced to dominate the reals in the generic extension via $\mathbb{P} \mid L_{\alpha}$ and, a fortiori, it will dominate $\dot{g}$.

Proposition 1.7 (Adjoining a mad family). Assume L has uncountable cofinality and $L_{\mathrm{mad}}$ is cofinal in $L$. Then $\mathbb{P}\left\lceil L\right.$ canonically adjoins a mad family of size $\left|L_{\mathrm{mad}}\right|$.

Proof. Let $G$ be $\mathbb{P} \nmid L$-generic over the ground model. For $x \in L_{\text {mad }}$ define $Y_{x}=$ $\{n \in \omega ; p(x)(n)=1$ for some $p \in G\}$. Let $\mathcal{A}=\left\{Y_{x} ; x \in L_{\text {mad }}\right\}$. By definition of the p.o., $\mathcal{A}$ is an almost-disjoint family. We need to check maximality. So let $\dot{Z}$ be a $\mathbb{P} \nmid L$-name for an infinite subset of $\omega$ and assume by way of contradiction that $p$ forces that $\dot{Z}$ is almost disjoint from all $\dot{Y}_{x}$. By Lemma 1.4 there is a countable set $A$ such that $p \in \mathbb{P} \mid \operatorname{cl}(A)$ and $\dot{Z}$ is a $\mathbb{P} \mid \mathrm{cl}(A)$-name. Since $L$ has uncountable cofinality and $L_{\text {mad }}$ is cofinal in $L$, there is $x \in L_{\text {mad }}$ such that $\operatorname{cl}(A) \subseteq L_{x}$. By Main Lemma 1.1 we know that $\mathbb{P}|\operatorname{cl}(A)<\circ \mathbb{P}| L_{x}<\circ \mathbb{P}\lceil L$.

Find $k_{0}$ and $p_{0} \leq_{\mathbb{P} \mid L} p$ such that

$$
p_{0} \Vdash_{\mathbb{P} \nmid L} \dot{Z} \cap \dot{Y}_{x} \subseteq k_{0} .
$$

Put $\bar{p}_{0}=p_{0}\left\lceil L_{x}\right.$. Clearly any $\dot{Y}_{y}, y \in \operatorname{dom}\left(\bar{p}_{0}\right) \cap L_{\text {mad }}$, is a $\mathbb{P}\left\lceil L_{x}\right.$-name. So we can find $k_{1} \geq k_{0}$ and $\bar{p}_{1} \leq \mathbb{P} \mid L_{x} \bar{p}_{0}$ such that

$$
\bar{p}_{1} \Vdash_{\mathbb{P} \nmid L_{x}} \dot{Z} \cap \dot{Y}_{y} \subseteq k_{1}
$$

for all $y \in \operatorname{dom}\left(\bar{p}_{0}\right) \cap L_{\text {mad }}$. Since $\dot{Z}$ is forced to be infinite, we can find $\bar{p}_{2} \leq_{\mathbb{P} \mid L_{x}} \bar{p}_{1}$ and $i_{0} \geq k_{1}$ such that $\bar{p}_{2} \Vdash_{\mathbb{P} \nmid L_{x}} i_{0} \in \dot{Z}$. Without loss of generality, $n^{\bar{p}_{2}}>i_{0}$. Then we must necessarily have $\bar{p}_{2}(y)\left(i_{0}\right)=0$ for all $y \in \operatorname{dom}\left(\bar{p}_{0}\right) \cap L_{\text {mad }}$.

Define a condition $p_{2}$ by

- $\operatorname{dom}\left(p_{2}\right)=\operatorname{dom}\left(\bar{p}_{2}\right) \cup \operatorname{dom}\left(p_{0}\right), n^{p_{2}}=n^{\bar{p}_{2}}$,

- $p_{2} \uparrow L_{x}=\bar{p}_{2}$,

- $p_{2}(z)=p_{0}(z)$ for all $z \in L_{\text {Hech }} \cap \operatorname{dom}\left(p_{0}\right), z>x$,

- $p_{2}(z) \supset p_{0}(z), p_{2}(z)(i)=0$ for all $i$ with $n^{p_{0}} \leq i<n^{p_{2}}$ and all $z \in$ $L_{\mathrm{mad}} \cap \operatorname{dom}\left(p_{0}\right), z>x$,

- $p_{2}(x) \supset p_{0}(x)$,

$$
p_{2}(x)(i)= \begin{cases}1 & \text { if } i=i_{0}, \\ 0 & \text { for all } i \neq i_{0} \text { with } n^{p_{0}} \leq i<n^{p_{2}} .\end{cases}
$$

It is straightforward to verify that $p_{2} \in \mathbb{P}\left\lceil L\right.$ and that $p_{2} \leq_{\mathbb{P} \nmid L} p_{0}$. Since

$$
p_{2} \Vdash_{\mathbb{P} \mid L} i_{0} \in \dot{Z} \cap \dot{Y}_{x},
$$

we have a contradiction. 


\section{BUILDing A TEMPlate FOR ADJOINING A MAD FAMILY}

For simplicity assume $\mathrm{CH}$ for the remainder of the paper.

Assume $\lambda_{0} \geq \aleph_{2}$ is regular, and $\lambda>\lambda_{0}$ is a singular cardinal of countable cofinality, say $\lambda=\bigcup_{n} \lambda_{n}$, the $\lambda_{n}$ being regular, equal to $\lambda_{n}^{\aleph_{0}}$, and strictly increasing. Also suppose $\kappa^{\aleph_{0}}<\lambda_{n}$ for $\kappa<\lambda_{n}$. As usual, $\mu^{*}$ denotes (a disjoint copy of) $\mu$ with the reverse ordering. Elements of $\mu$ will be called positive, and members of $\mu^{*}$ are negative. For each $n$ choose a partition $\lambda_{n}^{*}=\bigcup_{\alpha<\omega_{1}} S_{n}^{\alpha}$ such that each $S_{n}^{\alpha}$ is co-initial in $\lambda_{n}^{*}$. Also assume $S_{n}^{\alpha} \cap \lambda_{m}^{*}=S_{m}^{\alpha}$ for $m<n$.

The following definition is motivated by Shelah's work [S2].

Definition (Template for adjoining a mad family). Define $L=L(\lambda)$ as follows. Elements of $L$ are nonempty finite sequences $x$ (i.e., $\operatorname{dom}(x) \in \omega$ ) such that

- $x(0) \in \lambda_{0}$,

- $x(n) \in \lambda_{n}^{*} \cup \lambda_{n}$ for $0<n<|x|-1$, and

- in case $|x| \geq 2$, if $x(|x|-2)$ is positive, then $x(|x|-1) \in \lambda_{|x|-1}^{*} \cup \lambda$, and if $x(|x|-2)$ is negative, then $x(|x|-1) \in \lambda^{*} \cup \lambda_{|x|-1}$.

Say $x \in L_{\text {Hech }}$ if $|x|=1$ or $x(|x|-1) \in \lambda_{|x|-1}^{*} \cup \lambda_{|x|-1}$. Otherwise $x \in L_{\text {mad }}$. (This means that $x \in L_{\text {mad }}$ iff $|x| \geq 2$ and either $x(|x|-2)$ is positive and $x(|x|-1) \geq \lambda_{|x|-1}$ or $x(|x|-2)$ is negative and $\left.x(|x|-1) \leq \lambda_{|x|-1}^{*}.\right)$ Equip $L$ with the following lexicographic-like ordering: $x<y$ iff

- either $x \subset y$ and $y(|x|)$ is positive,

- or $y \subset x$ and $x(|y|)$ is negative,

- or, letting $n:=\min \{m: x(m) \neq y(m)\}$, either $x(n)$ is negative and $y(n)$ is positive, or both are positive and $x(n)<_{\lambda} y(n)$, or both are negative and $x(n)<_{\lambda^{*}} y(n)$ (i.e., there are $\alpha<\beta<\lambda$ such that $x(n)=\beta^{*}<_{\lambda^{*}} \alpha^{*}=$ $y(n))$.

It is immediate that this is indeed a linear ordering.

We identify sequences of length one with their ranges so that $\lambda_{0}$ is a cofinal subset of $L$. Say $x \in L_{\mathrm{Hech}}$ is relevant if $|x| \geq 3$ is odd, $x(n)$ is negative for odd $n$ and positive for even $n, x(|x|-1)<\omega_{1}$, and whenever $n<m$ are even such that $x(n), x(m)<\omega_{1}$, then there are $\beta<\alpha$ such that $x(n-1) \in S_{n-1}^{\alpha}$ and $x(m-1) \in S_{m-1}^{\beta}$. For relevant $x$, set $J_{x}=[x \uparrow(|x|-1), x)$, the interval of nodes between $x \uparrow(|x|-1)$ and $x$ in the order of $L$. Notice that if $x<y$ are relevant, then either $J_{x} \cap J_{y}=\emptyset$ or $J_{x} \subset J_{y}$ (in which case we also have $|y| \leq|x|$, $x \uparrow(|y|-1)=y\lceil(|y|-1)$ and $x(|y|-1) \leq y(|y|-1))$.

Define $\mathcal{I}=\mathcal{I}(\lambda)$ to be the collection of all finite unions of sets of the form

- $L_{\alpha}$ for $\alpha \leq \lambda_{0}$,

- $\mathrm{cl}\left(J_{x}\right)$ for relevant $x$,

- $\operatorname{cl}(\{x\})$ for $x \in L_{\text {Hech }}$, and

- $L_{x} \cap L_{\text {mad }}$ for $x \in L_{\text {Hech. }}$

So $L(\lambda)$ is a subtree of $\left(\lambda^{*} \cup \lambda\right)^{<\omega}$ (i.e., it is closed under taking initial segments). The nodes belonging to $L_{\text {mad }}$ are exactly the terminal (= maximal) nodes of this tree. The point of the $J_{x}$ is that we need "copies" of the large supports given by the $L_{\alpha}$ for isomorphism-of-names arguments. The $S_{n}^{\alpha}$, then, are used to code the places where we put the $J_{x}$ so that we basically get well-foundedness for free.

Lemma 2.1. $(L, \mathcal{I})$ is a template. 
Proof. Clauses (1) and (6) in the definition of template are immediate, as is closure under finite unions. To see closure under finite intersections, it suffices to argue that the intersection of any two sets of the above form (i.e., $L_{\alpha}, \operatorname{cl}\left(J_{x}\right), \operatorname{cl}(\{x\})$, and $\left.L_{x} \cap L_{\text {mad }}\right)$ is again of this form. This, however, is straightforward so that (2) holds as well.

To prove $\left(3^{\prime}\right)$, let $x \in L_{\mathrm{Hech}}$ and $y \in L_{x}$. In case $y \in L_{\mathrm{Hech}}$, we have $y \in \operatorname{cl}(\{y\}) \subseteq$ $L_{x}$. If $y \in L_{\text {mad }}, y \in L_{x} \cap L_{\text {mad }} \subseteq L_{x}$.

For $\left(4^{\prime}\right)$, it suffices again to consider sets $A$ from $\mathcal{I}$ of the above form. Let $x \in L_{\text {Hech }} \backslash A$. Without loss of generality, $A \backslash L_{x} \neq \emptyset$. If $A$ is of the form $L_{y}$, $\mathrm{cl}\left(J_{y}\right), \operatorname{cl}(\{y\})$ or $L_{y} \cap L_{\mathrm{mad}}$, then we must have $y>x . A=L_{y}$ is impossible and if $A=\mathrm{cl}\left(J_{y}\right)$, then $x<y \uparrow(|y|-1)=\min \left(J_{y}\right)$. So, in each of the possible cases, the intersection with $L_{x}$ is $L_{x} \cap L_{\text {mad }}$.

We are left with showing well-foundedness $\left(5^{\prime}\right)$. Assume $A_{n}, n \in \omega$, is a de-

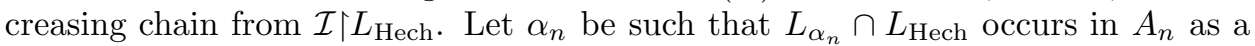
component. Choose $\alpha_{n_{0}}$ minimal among the $\alpha_{n}$. Without loss of generality, $n_{0}=0$. Then all $L_{\alpha_{n}} \cap L_{\text {Hech }}$ are the same and it suffices to consider the $J_{x}$-components. Thus we may assume, without loss of generality, that $A_{0}=J_{x_{0}^{(〉}} \cap L_{\mathrm{Hech}}$, and there is a finitely-branching tree $T \subseteq \omega^{<\omega}$ such that $A_{n}=\left(\bigcup_{\sigma \in T \cap \omega^{n}} J_{x_{n}^{\sigma}} \cup F_{n}\right) \cap L_{\text {Hech }}$ where the $F_{n} \subseteq L_{\mathrm{Hech}}$ are finite, and such that $\sigma \subseteq \tau,|\sigma|=n<|\tau|=m$, implies $J_{x_{m}^{\tau}} \subseteq J_{x_{n}^{\sigma}}$, and such that the $J_{x_{n}^{\sigma}}, \sigma \in T \cap \omega^{n}$, are pairwise disjoint. Now note that if $f \in[T]$ is a branch, then the sequence $\left\{x_{n}^{f \nmid n} ; n \in \omega\right\}$ must eventually stabilize. (First argue that if $\left|x_{n}^{f \nmid n}\right| \rightarrow \infty$, then $\left\{\alpha ; x_{n}^{f \nmid n}\left(\left|x_{n}^{f \nmid n}\right|-2\right) \in S_{n}^{\alpha}\right.$ for some $n\}$ would constitute a decreasing sequence of ordinals. Then notice that if $\left|x_{n}^{f \nmid n}\right|$ is eventually constant, so is the decreasing sequence $x_{n}^{f \nmid n}\left(\left|x_{n}^{f \nmid n}\right|-1\right)$.) Since $T$ is a finitely-branching tree this means that the total number of the $x_{n}^{\sigma}$ is finite which in turn entails that the sequence of the $A_{n}$ eventually stabilizes.

Corollary 2.2 (Bounds for $\mathfrak{a}$ ). $\mathbb{P} \nmid L$ forces $\mathfrak{b}=\mathfrak{d}=\lambda_{0}$ and adjoins a mad family of size $\lambda$ (so that $\lambda_{0} \leq \mathfrak{a} \leq \lambda$ ).

Proof. This is immediate by construction of $\mathbb{P}\lceil L$ and Propositions 1.6 and 1.7

\section{Killing mad FAMilies USING TEMPlates}

We are left with showing there is no mad family of size less than $\lambda$ in the generic extension. As explained in the Introduction, this is an (albeit sophisticated) isomorphism-of-names argument. Isomorphisms of names canonically boil down to certain brands of partial isomorphisms between subsets of $L$, and we begin with their investigation.

Definition (Isomorphism). Let $A, B \subseteq L$ be countable trees 8 Call $A$ and $B$ isomorphic $(A \cong B)$ iff there is a bijection $\phi=\phi_{A, B}: A \rightarrow B$ such that

(a) $|\phi(x)|=|x|$,

(b) $\phi(x)\lceil n=\phi(x\lceil n)$,

(c) $x<y$ iff $\phi(x)<\phi(y)$,

(d) $x(n)$ is positive iff $\phi(x)(n)$ is positive,

(e) $x \in L_{\text {mad }}$ iff $\phi(x) \in L_{\text {mad }}$

for all $x, y \in A$ and all $n \in \omega$, and such that

\footnotetext{
${ }^{8}$ Recall $A$ is a tree if it is closed under taking initial segments, i.e., given $x \in A$, we have $x\lceil n \in A$ for all $n \in \omega$.
} 
(f) $\mathcal{I}\lceil A$ is mapped to $\mathcal{I}\lceil B$ via $\phi$.

Since the trace of $\mathcal{I}$ on each countable set is countable, there are at most $2^{\aleph_{0}}=\aleph_{1}$ isomorphism types.

This, the strongest notion of "isomorphism" we shall consider, will be used in several pruning arguments below. However, for most purposes the following is sufficient.

Definition (Weak isomorphism). Let $A, B \subseteq L$ be arbitrary. We say that $A$ and $B$ are weakly isomorphic $\left(A \cong \cong_{\text {weak }} B\right)$ if (e) is satisfied and instead of clauses (c), (f) we have

$\left(\mathrm{c}^{\prime}\right) x<y$ iff $\phi(x)<\phi(y)$ for all $x, y$ such that there is $z \in L_{\mathrm{Hech}} \cap A$ with $x \leq z \leq y$,

and

$\left(\mathrm{f}^{\prime}\right) \phi$ maps a cofinal subset of $\mathcal{I}\lceil A$ to a cofinal subset of $\mathcal{I}\lceil B$, respectively.

Lemma 3.1. Let $A$ and $B$ be countable trees such that $L_{\mathrm{Hech}} \cap A$ ( $L_{\mathrm{Hech}} \cap B$, respectively) is cofinal in $A$ (in $B$, resp.). If $A \cong B$, as witnessed by $\phi$, then there is $\psi$ extending $\phi$ and witnessing that $\mathrm{cl}(A) \cong_{\text {weak }} \mathrm{cl}(B)$.

Proof. Call a nonempty $X \subseteq \mathrm{cl}(A) \cap L_{\text {mad }}$ connected if given $x<y$ from $X$, the interval $[x, y]$ is disjoint from $A \cap L_{\text {Hech }}$. A maximal connected set is called a connected component. Note every connected component has size $\lambda$ (because $L_{\mathrm{Hech}}$ is cofinal in $A$ ) and $\operatorname{cl}(A) \cap L_{\text {mad }}$ is a disjoint union of at most countably many connected components.

Given $x \in L_{\mathrm{Hech}} \cap A$ with $x(|x|-1)$ being positive, put Comp $x=\left\{y \in L_{\mathrm{mad}} ; y<\right.$ $z$ for every $z \in A \cap L_{\text {Hech }}$ with $z \supseteq x$ and $y>z$ for every $z \in A \cap L_{\text {Hech }}$ such that $z|| x \mid<x\}$. Clearly Comp $\mathrm{p}_{x}$ is a connected component. Dually, define Comp $\mathrm{p}_{x}$ for $x \in L_{\text {Hech }} \cap A$ with negative $x(|x|-1)$ by interchanging $<$ and $>$.

For each $y \in \operatorname{cl}(A) \cap L_{\text {mad }}$, there is $x \in L_{\text {Hech }} \cap A$ with $y \in \operatorname{Comp}_{x}$. To see this, let $n<|y|$ be maximal such that $y\lceil n \in A$. Assume, without loss of generality, $y(n)$ is positive. Let $k \leq n$ be minimal such that all $y(i)$ for $k \leq i \leq n$ are positive. If possible choose $m, k \leq m \leq n$, and $x \in A \cap L_{\mathrm{Hech}},|x|=m+1$, such that $x\lceil m=y\lceil m, x(m)>y(m)$ is minimal, and such that $m$ is the maximal value for which such an $x$ can be found. Then $y \in$ Comp $_{x}$. If $m$ and $x$ cannot be found, we let $x=y\left\lceil k\right.$ and check $y \in$ Comp $_{x}$ (note that $x(|x|-1)=y(k-1)$ is negative in this case so that the second alternative of the definition of Comp applies). Therefore $\mathrm{cl}(A) \cap L_{\text {mad }}=\bigcup_{x \in L_{\mathrm{Hech}} \cap A} \operatorname{Comp}_{x}$.

Also notice that for $x, x^{\prime} \in L_{\mathrm{Hech}} \cap A$, if $\mathrm{Comp}_{x}=\mathrm{Comp}_{x^{\prime}}$, then $\operatorname{Comp}_{\phi(x)}=$ $\operatorname{Comp}_{\phi\left(x^{\prime}\right)}$, and if Comp ${ }_{x} \cap \operatorname{Comp}_{x^{\prime}}=\emptyset$, then $\operatorname{Comp}_{\phi(x)} \cap \operatorname{Comp}_{\phi\left(x^{\prime}\right)}=\emptyset$.

So we can simply extend $\phi$ to $\psi$ by mapping $\operatorname{Comp}_{x}$ to $\operatorname{Comp}_{\phi(x)}$ for all $x \in$ $L_{\text {Hech }} \cap A$. Then $\left(\mathrm{c}^{\prime}\right)$ and (e) are immediate. To see $\left(\mathrm{f}^{\prime}\right)$, note that, by definition of the template, sets in $\mathcal{I}\lceil\mathrm{cl}(A)$ that are unions of sets from $\mathcal{I}\lceil A$ and of sets of the form $L_{x} \cap L_{\text {mad }}$ are cofinal in $\mathcal{I}\lceil\operatorname{cl}(A)$. However, since $\phi$ identifies sets of $\mathcal{I}\lceil A$ and sets of $\mathcal{I}\lceil B, \psi$ identifies sets of the latter kind.

Note that we did not use the full strength of our notion of isomorphism in the above proof. Clauses (c) and (f) could be replaced by $\left(c^{\prime}\right)$ and $\left(f^{\prime}\right)$ respectively. Furthermore, instead of dealing with trees $A$ and $B$ (and having (a), (b), and (d)), 
it suffices that $\operatorname{cl}(A) \cap L_{\text {mad }}$ is the union of the components $\operatorname{Comp}_{x}, x \in L_{\mathrm{Hech}} \cap A$, and similarly for $B$, and that extending $\phi$ by mapping Comp ${ }_{x}$ to $\operatorname{Comp}_{\phi(x)}$ preserves $\left(c^{\prime}\right)$.

Lemma 3.2. If $A \cong_{\text {weak }} B$, then $\mathbb{P}\lceil A \cong \mathbb{P}\lceil B$.

Proof. Notice that clauses (c), (e) and (f) are enough to guarantee that $\mathbb{P}\lceil A \cong$ $\mathbb{P}\left\lceil B\right.$. By Lemma 1.5, this is still true if (f) is replaced by $\left(\mathrm{f}^{\prime}\right)$. Finally, by the way $\mathbb{P}\left\lceil A\right.$ is defined recursively, interchanging elements of $L_{\text {mad }}$ that belong to the same connected component of $A \cap L_{\text {mad }}$ does not affect the p.o 9 (because the interchanging map sends a cofinal subset of $\mathcal{I}\lceil A$ to a cofinal subset of $\mathcal{I}\lceil A$, see 1.5 $)$.

Completion of the proof of the Main Theorem. Now assume $\dot{\mathcal{A}}$ is a name for an almost-disjoint family of size $<\lambda$, say $\dot{\mathcal{A}}$ is listed as $\left\{\dot{A}^{\alpha} ; \alpha<\kappa\right\}$. Also assume $\dot{\mathcal{A}}$ is forced to have size at least $\lambda_{0}$. Let $k<\omega$ be maximal such that $\kappa \geq \lambda_{k}$. Without loss of generality, $\kappa \geq \lambda_{k} \cdot 2$. We shall perform several standard pruning arguments, reordering the family of the $\dot{A}^{\alpha}$ so that the first $\lambda_{k}$ many look very "similar", that is, those $\dot{A}^{\alpha}$ that do not fit the pattern get removed to higher indices. This is why we stipulate $\kappa \geq \lambda_{k} \cdot 2$. Eventually, the first $\omega_{1}$ many $\dot{A}^{\alpha}$ will suffice, and it is those that we use to create a new name $\dot{A}^{\kappa}$ witnessing non-maximality.

For fixed $\alpha$, find countable maximal antichains $\left\{p_{n, i}^{\alpha} ; i \in \omega\right\} \subseteq \mathbb{P} \mid L, n \in \omega$, and $\left\{k_{n, i}^{\alpha} \in 2 ; i, n \in \omega\right\}$ such that $p_{n, i}^{\alpha} \Vdash n \in \dot{A}^{\alpha}$ iff $k_{n, i}^{\alpha}=1$ and $p_{n, i}^{\alpha} \Vdash n \notin \dot{A}^{\alpha}$ iff $k_{n, i}^{\alpha}=0$. Let $B^{\alpha}=\bigcup\left\{\operatorname{dom}\left(p_{n, i}^{\alpha}\right) ; i, n \in \omega\right\} \subseteq L$. $B^{\alpha}$ is at most countable. Without loss of generality, it is a tree. Let $C^{\alpha}=\operatorname{cl}\left(B^{\alpha}\right)$. Put $B:=\bigcup_{\alpha} B^{\alpha}$. So $|B|<\lambda_{k+1}<\lambda$. By $C H$ and the $\Delta$-system lemma we may assume, without loss of generality, that the $\left\{B^{\alpha} ; \alpha<\lambda_{k}\right\}$ form a $\Delta$-system, and that the bijection $\phi=\phi^{\alpha, \beta}$ (see above) sending $B^{\alpha}$ to $B^{\beta}$ is an isomorphism fixing the root $R$ of the system. Because there are only $\lambda_{k-1}^{\aleph_{0}}=\lambda_{k-1}$ many countable subsets of $L_{\mathrm{Hech}} \cap\left(\lambda^{*} \cup \lambda\right)^{k}$, we may also assume that if $x \in B^{\alpha} \cap L_{\mathrm{Hech}}$ and $|x| \leq k$, then $x \in R$. Also stipulate that there is some $\theta_{0}<\omega_{1}$ such that whenever $\alpha<\lambda_{k}, x \in B^{\alpha}, j$ odd and $x(j) \in \lambda_{j}^{*}$, then $x(j) \in S_{j}^{\theta}$ for some $\theta<\theta_{0}$. As explained above, $\phi$ canonically induces a weak isomorphism $\psi=\psi^{\alpha, \beta}$ between $C^{\alpha}$ and $C^{\beta}$ (Lemma 3.1), which in turn yields an isomorphism $\chi=\chi^{\alpha, \beta}$ between $\mathbb{P}\left\lceil C^{\alpha}\right.$ and $\mathbb{P}\left\lceil C^{\beta}\right.$ (Lemma 3.2) both of which embed into $\mathbb{P}\left\lceil L\right.$ (Main Lemma 1.1), as well as between $\mathbb{P}\left\lceil C^{\alpha}\right.$-names and $\mathbb{P}\left\lceil C^{\beta}\right.$-names. Furthermore, since connected components are homogeneous from the forcing point of view, since $C^{\alpha} \cap L_{\mathrm{Hech}}=B^{\alpha} \cap L_{\mathrm{Hech}}$ is countable, and since $C^{\alpha} \cap L_{\text {mad }}$ has only countably many connected components (see the proofs of Lemmas 3.1 and 3.2), it has, up to isomorphism, only $2^{\aleph_{0}}=\aleph_{1}$ many isomorphism types of names. (Of course, there are a total of $\lambda^{\aleph_{0}}$ names.) Therefore, we may also suppose that $\chi$ identifies $\dot{A}^{\alpha}$ with $\dot{A}^{\beta}$, which means, more explicitly, that $k_{n, i}:=k_{n, i}^{\alpha}=k_{n, i}^{\beta}$ and $\chi\left(p_{n, i}^{\alpha}\right)=p_{n, i}^{\beta}$.

Write $B^{\alpha}=\left\{x_{s}^{\alpha} ; s \in T\right\}$ where $T \subseteq\left(\omega_{1}^{*} \cup \omega_{1}\right)^{<\omega}$ is the canonical tree isomorphic to any $B^{\alpha}$. This means in particular that $\phi^{\alpha, \beta}\left(x_{s}^{\alpha}\right)=x_{s}^{\beta}$, that $|s|=\left|x_{s}^{\alpha}\right|$, and that $s(n)$ is positive iff $x_{s}^{\alpha}(n)$ is positive. Let $T_{\mathrm{Hech}}=\left\{s \in T ; x_{s}^{\alpha} \in L_{\mathrm{Hech}}\right\}$ and $T_{\text {mad }}=\left\{s \in T ; x_{s}^{\alpha} \in L_{\text {mad }}\right\}$. $T_{\text {Hech }}$ is a subtree of $T$, while $T_{\text {mad }}$ is a set of terminal nodes of $T$. Furthermore, let $S \subseteq T$ be the subtree of $T$ corresponding to the root,

${ }^{9}$ That is, the order structure on connected components of $A \cap L_{\mathrm{mad}}$ is irrelevant, and connected components are homogeneous from the forcing point of view. 
that is, $s \in S$ iff $x_{s}^{\alpha} \in R$ for all $\alpha$. So, for $\alpha \neq \beta, x_{s}^{\alpha}=x_{s}^{\beta}$ iff $s \in S$. Furthermore, if $s \in T_{\text {Hech }} \backslash S$, then $|s| \geq k+1$. List $\{t \in T \backslash S ; t \uparrow(|t|-1) \in S\}=\left\{t_{n} ; n \geq 1\right\}$. For $\alpha<\beta$ define

$F(\{\alpha, \beta\})= \begin{cases}\min \{n ; & \text { either } t_{n}\left(\left|t_{n}\right|-1\right) \in \omega_{1} \text { and } x_{t_{n}}^{\alpha}\left(\left|t_{n}\right|-1\right)>x_{t_{n}}^{\beta}\left(\left|t_{n}\right|-1\right) \\ & \left.\text { or } t_{n}\left(\left|t_{n}\right|-1\right) \in \omega_{1}^{*} \text { and } x_{t_{n}}^{\alpha}\left(\left|t_{n}\right|-1\right)<x_{t_{n}}^{\beta}\left(\left|t_{n}\right|-1\right)\right\} \\ & \text { if such an } n \text { exists, } \\ 0 & \text { otherwise. }\end{cases}$

Note that for each $n \geq 1$, every subset of $\lambda_{k}$ homogeneous in color $n$ must be finite. Using partition calculus as well as standard pruning arguments, we may therefore assume that for all $\alpha<\omega_{1}$, if $s \in S$ and $\hat{s}^{\wedge}\langle\zeta\rangle \notin S$, then

- if $\zeta$ is positive, then $x_{s^{\wedge}\langle\zeta\rangle}^{\alpha}(|s|)<x_{s^{\wedge}\langle\zeta\rangle}^{\beta}(|s|)$ for all $\alpha<\beta$, and all $x_{s^{\wedge}\langle\zeta\rangle}^{\alpha}(|s|)$ are larger than $\omega_{1}$, and if $\zeta<\xi, s^{\wedge}\langle\zeta\rangle, s^{\wedge}\langle\xi\rangle \notin S$, then

- either for all $\alpha, \beta$, we have $x_{s^{\wedge}\langle\zeta\rangle}^{\alpha}(|s|)<x_{s^{\wedge}\langle\xi\rangle}^{\beta}(|s|)$

(this is the case when $\sup _{\alpha} x_{s^{\wedge}\langle\zeta\rangle}^{\alpha}(|s|)<\sup _{\alpha} x_{s^{\wedge}\langle\xi\rangle}^{\alpha}(|s|)$ ),

- or for all $\alpha<\beta$, we have $x_{s^{\wedge}\langle\xi\rangle}^{\alpha}(|s|)<x_{s^{\wedge}\langle\zeta\rangle}^{\beta}(|s|)$

(this is the case when $\left.\sup _{\alpha} x_{s^{\wedge}\langle\zeta\rangle}^{\alpha}(|s|)=\sup _{\alpha} x_{s^{\wedge}\langle\xi\rangle}^{\alpha}(|s|)\right)$,

- if $\zeta$ is negative, then $x_{s^{\wedge}\langle\zeta\rangle}^{\alpha}(|s|)>x_{s^{\wedge}\langle\zeta\rangle}^{\beta}(|s|)$ for all $\alpha<\beta$, and if $\zeta<\xi$, $s^{\wedge}\langle\zeta\rangle, s^{\wedge}\langle\xi\rangle \notin S$, then

- either for all $\alpha, \beta$, we have $x_{s^{\wedge}\langle\zeta\rangle}^{\alpha}(|s|)>x_{s^{\wedge}\langle\xi\rangle}^{\beta}(|s|)$,

- or for all $\alpha<\beta$, we have $x_{s^{\wedge}\langle\xi\rangle}^{\alpha}(|s|)>x_{s^{\wedge}\langle\zeta\rangle}^{\beta}(|s|)$.

Define $x_{s}^{\kappa} \in L$ by recursion on the length of $s \in T$ as follows. If $s \in S$, then $x_{s}^{\kappa}=x_{s}^{\alpha}$ (a fortiori $\left.\left|x_{s}^{\kappa}\right|=\left|x_{s}^{\alpha}\right|=|s|\right)$ ). If $s \in S$ and $s^{\wedge}\langle\zeta\rangle \in T_{\text {Hech }} \backslash S$, let $x_{s^{\wedge}\langle\zeta\rangle}^{\kappa}(|s|)$ be the limit of the $x_{s^{\wedge}\langle\zeta\rangle}^{\alpha}(|s|)$ (so it is either the sup or the inf, depending on whether $\zeta$ is positive or negative). Next find $\gamma_{s}<\lambda_{|s|+1}, \gamma_{s}>\omega_{1}$ and $\gamma_{s}^{*} \in S_{|s|+1}^{\theta_{0}}$, such that for all such $s$ and $\zeta$,

- if $x_{s^{\wedge}\langle\zeta\rangle}^{\kappa}(|s|)=\sup _{\alpha} x_{s^{\wedge}\langle\zeta\rangle}^{\alpha}(|s|)$, then for all $y \in B$ with $y \uparrow(|s|+1)=$ $x_{s^{\wedge}\langle\zeta\rangle}^{\kappa}\left\lceil(|s|+1)\right.$, we have $y(|s|+1)>\gamma_{s}^{*}$,

- if $x_{s^{\wedge}\langle\zeta\rangle}^{\kappa}(|s|)=\inf _{\alpha} x_{s^{\wedge}\langle\zeta\rangle}^{\alpha}(|s|)$, then for all $y \in B$ with $y\lceil(|s|+1)=$ $x_{s^{\wedge}\langle\zeta\rangle}^{\kappa}\left\lceil(|s|+1)\right.$, we have $y(|s|+1)<\gamma_{s}$.

It is clear that we can find such $\gamma_{s}$ 's because $\lambda_{|s|+1}>|B|$ is regular (since $|s| \geq k$ ). In the first case, let $x_{s^{\wedge}\langle\zeta\rangle}^{\kappa}(|s|+1)=\gamma_{s}^{*}$. In the second case, let $x_{s^{\wedge}\langle\zeta\rangle}^{\kappa}(|s|+1)=\gamma_{s}$. To complete the definition of $x_{s^{\wedge}\langle\zeta\rangle}^{\kappa}$, stipulate $\left|x_{s^{\wedge}\langle\zeta\rangle}^{\kappa}\right|=\left|x_{s^{\wedge}\langle\zeta\rangle}^{\alpha}\right|+2=|s|+3$, and define

$$
x_{s^{\wedge}\langle\zeta\rangle}^{\kappa}(|s|+2)= \begin{cases}x_{s^{\wedge}\langle\zeta\rangle}^{0}(|s|) & \text { if }|s|>0 \\ \zeta & \text { if }|s|=0\end{cases}
$$

If $s \in S$ and $s^{\wedge}\langle\zeta\rangle \in T_{\text {mad }} \backslash S$, find $\gamma \in \lambda^{*} \cup \lambda$ such that $x_{s}^{\kappa^{\wedge}}\langle\gamma\rangle \in L_{\text {mad }}$ and for all $y \in B$ with $y$ I|s| $=x_{s}^{\kappa}$, we have $y(|s|) \neq \gamma$. Such $\gamma$ clearly exists because $\lambda>|B|$. Stipulate $\left|x_{s^{\wedge}\langle\zeta\rangle}^{\kappa}\right|=\left|x_{s^{\wedge}\langle\zeta\rangle}^{\alpha}\right|=|s|+1$ and let $x_{s^{\wedge}\langle\zeta\rangle}^{\kappa}(|s|)=\gamma$. Finally, for the remaining $t \in T$, stipulate again $\left|x_{t}^{\kappa}\right|=\left|x_{t}^{\alpha}\right|+2=|t|+2$, find $s \subset t$ with $s \in S$ maximal, and put $x_{t}^{\kappa} \uparrow(|s|+3)=x_{s^{\wedge}\langle t(|s|)\rangle}^{\kappa}$ and $x_{t}^{\kappa}(j+2)=x_{t}^{0}(j)$ for $j>|s|$.

Let $B^{\kappa}=\left\{x_{s}^{\kappa} ; s \in T\right\}$. Notice that $B^{\kappa}$, although very tree-like, is not a tree like the $B^{\alpha}$ 's. We proceed to verify that $\left(B^{\kappa}, \mathcal{I}\left\lceil B^{\kappa}\right)\right.$ and $\left(B^{\alpha}, \mathcal{I}\left\lceil B^{\alpha}\right), \alpha<\omega_{1}\right.$, are weakly isomorphic (clearly (a), (b) and (d) will fail but this is not relevant for us). 
Fix $\alpha$ and define $\phi=\phi^{\alpha, \kappa}$ by $\phi\left(x_{s}^{\alpha}\right)=x_{s}^{\kappa}$. Without loss of generality, $\alpha=0$. $\left(\mathrm{c}^{\prime}\right)$ and (e) are immediate by construction. So let us check that the trace of $\mathcal{I}$ on $B^{0}$ is mapped to a cofinal subset of $\mathcal{I}\left\lceil B^{\kappa}\right.$. First fix $\beta$ and consider $L_{\beta}$. Notice that there is $\beta_{0} \leq \beta$ such that $\phi\left(L_{\beta_{0}} \cap B^{0}\right)=L_{\beta_{0}} \cap B^{\kappa}=L_{\beta} \cap B^{\kappa}$. For any $s \in T$ with $x_{s}^{0} \in L_{\beta}$ yet $x_{s}^{\kappa} \notin L_{\beta}$ one must have $x_{s}^{\kappa}(0)>\beta \geq x_{s}^{0}(0) \geq \beta_{0}$ and $x_{s}^{\kappa}(0)$ is an $\omega_{1}$-limit of the $x_{s}^{\alpha}(0)$ 's. In particular, for all such $s, x_{s}^{\kappa}(0)$ must have the same value, say $\gamma_{0}$. Also $x_{s}^{\kappa}(1)=\gamma_{\langle\rangle}^{*}$ and $x_{s}^{\kappa}(2)=s(0)<\omega_{1}$. This means, however, that $L_{\beta} \cap B^{0}$ is mapped to $\left(L_{\beta} \cup \mathrm{cl}\left(J_{x}\right)\right) \cap B^{\kappa}$ via $\phi$ where $|x|=3, x(0)=\gamma_{0}, x(1)=\gamma_{\langle\rangle}^{*}$ and $x(2)=\sup \left\{s(0)+1 ; x_{s}^{0}(0)<\beta\right\}$. Next assume $x$ is relevant and consider $\mathrm{cl}\left(J_{x}\right)$. Assume $J_{x} \cap B^{0} \neq \emptyset$. Then there is $s \in T$ such that $|s|=|x|-1$ and $x_{s}^{0}=x \uparrow(|x|-1)$. In case $s \in S$, we have $x_{s}^{\kappa}=x_{s}^{0}$ and $J_{x} \cap B^{0}$ is mapped to $J_{x} \cap B^{\kappa}$ via $\phi$ because $y \in R$ for any $y \in B^{0}$ with $|y|=|x|, y \uparrow(|x|-1)=x_{s}^{0}$ and $y(|x|-1) \leq x(|x|-1)$. In case $s \in T \backslash S$, let $j_{0}<|s|$ be maximal with $s\left\lceil j_{0} \in S\right.$. Define $y$ by $|y|=|x|+2$, $y \uparrow(|y|-1)=x_{s}^{\kappa}$ and $y(|y|-1)=x(|x|-1)$ and note that $J_{x} \cap B^{0}$ gets mapped to $J_{y} \cap B^{\kappa}$ provided we can show $y$ is relevant. In case $j_{0}>0$ there is nothing to show because whenever $x_{s}^{0}(j)>\omega_{1}, j \geq j_{0}$ even, then also $x_{s}^{\kappa}(j+2)=x_{s}^{0}(j)>\omega_{1}$, and, if $j_{0}$ is even, we additionally have $x_{s}^{\kappa}\left(j_{0}\right)=\sup _{\alpha} x_{s}^{\alpha}\left(j_{0}\right)>\omega_{1}$ while, if $j_{0}$ is odd, we additionally have $x_{s}^{\kappa}\left(j_{0}+1\right)=\gamma_{s\left\lceil j_{0}\right.}>\omega_{1}$. In case $j_{0}=0$ this is true because $x_{s}^{\kappa}(1) \in S_{1}^{\theta_{0}}$ and $\theta_{0}$ is larger than all the $\theta$ for which $x_{s}^{\kappa}(j) \in S_{j}^{\theta}$ where $j>1$ is odd.

Even though $B^{\kappa}$ is not a tree, we can verify, as in the proof of Lemma 3.1, that, letting $C^{\kappa}:=\operatorname{cl}\left(B^{\kappa}\right), C^{\kappa} \cap L_{\mathrm{mad}}=\bigcup_{s \in T} \operatorname{Comp}_{x_{s}^{\kappa}}$ and that $\psi=\psi^{\alpha, \kappa}: C^{\alpha} \rightarrow C^{\kappa}$, $\alpha<\omega_{1}$, which extends $\phi$ and maps Comp $x_{s}^{\alpha}$ to Comp $x_{x_{s}^{\kappa}}$ is a weak isomorphism.

By Lemma 3.2 $\mathbb{P}\left\lceil C^{\alpha}\right.$ and $\mathbb{P} \mid C^{\kappa}$ are isomorphic by a map $\chi=\chi^{\alpha, \kappa} . \quad \chi$ sends $\mathbb{P}\left\lceil C^{\alpha}\right.$-names to $\mathbb{P}\left\lceil C^{\kappa}\right.$-names, and we define $\dot{A}^{\kappa}$ to be the image of $\dot{A}^{\alpha}$ under $\chi$.

By construction, it is then also immediate that whenever $\beta<\kappa$, we can find $\alpha<\omega_{1}$ such that $B^{\kappa} \cup B^{\beta}$ and $B^{\alpha} \cup B^{\beta}$ are weakly isomorphic via the mapping fixing nodes of $B^{\beta}$ and sending the $x_{s}^{\kappa}$ to the corresponding $x_{s}^{\alpha}$, and such that this mapping identifies cofinal subsets of the traces of $\mathcal{I}$ on the two sets 10 Again, this weak isomorphism canonically extends to a weak isomorphism of $C^{\kappa} \cup C^{\beta}$ and $C^{\alpha} \cup C^{\beta}$, which in turn means that $\mathbb{P}\left\lceil C^{\kappa} \cup C^{\beta}\right.$ and $\mathbb{P}\left\lceil C^{\alpha} \cup C^{\beta}\right.$ are isomorphic (Lemma 3.2) by a mapping sending the name $\dot{A}^{\kappa}$ to $\dot{A}^{\alpha}$. Since $\dot{A}^{\alpha}$ and $\dot{A}^{\beta}$ are forced to be almost disjoint (by $\mathbb{P}\left\lceil C^{\alpha} \cup C^{\beta}\right.$ ), so are $\dot{A}^{\kappa}$ and $\dot{A}^{\beta}$ (by the isomorphic $\mathbb{P}\left\lceil C^{\kappa} \cup C^{\beta}\right.$ ). Since $\mathbb{P}\left\lceil C^{\kappa} \cup C^{\beta}\right.$ embeds into $\mathbb{P}\lceil L$ (Lemma 1.1), this completes the proof of the non-maximality of $\dot{\mathcal{A}}$ and, by Corollary 2.2 of the Main Theorem.

\section{REFERENCES}

[BJ] T. Bartoszyński and H. Judah, Set Theory. On the structure of the real line, A K Peters, Wellesley, MA, 1995. MR 96k:03002

[Bl] A. Blass, Combinatorial cardinal characteristics of the continuum, in: Handbook of Set Theory (A. Kanamori et al., eds.), to appear.

[Br] J. Brendle, Mad families and iteration theory, in: Logic and Algebra (Y. Zhang, ed.), Contemp. Math. 302 (2002), Amer. Math. Soc., Providence, RI, 1-31.

[H1] S. Hechler, Short complete nested sequences in $\beta \mathbb{N} \backslash \mathbb{N}$ and small maximal almost-disjoint families, General Topology and Appl. 2 (1972), 139-149. MR 46:7028

[H2] S. Hechler, On the existence of certain cofinal subsets of $\omega^{\omega}$, in: Axiomatic Set Theory (T. Jech, ed.), Proc. Sympos. Pure Math. 13 (1974), 155-173. MR 50:12716

[M] A. Miller, Arnie Miller's problem list, in: Set Theory of the Reals (H. Judah, ed.), Israel Math. Conf. Proc. 6 (1993), 645-654. MR 94m:03073

\footnotetext{
${ }^{10}$ In fact, this is true for all but countably many $\alpha<\omega_{1}$.
} 
[S1] S. Shelah, Covering of the null ideal may have countable cofinality, Fund. Math. 166 (2000), 109-136. (publication number 592) MR 2001m:03101

[S2] S. Shelah, Are a and $\mathbb{D}$ your cup of tea? Acta Math., to appear. (publication number 700)

[vD] E. van Douwen, The integers and topology, in: Handbook of Set-theoretic Topology (K. Kunen and J. Vaughan, eds.), North-Holland, Amsterdam (1984), 111-167. MR 87f:54008

[V] J. E. Vaughan, Small uncountable cardinals and topology, in: Open Problems in Topology (J. van Mill and G. M. Reed, eds.), North-Holland (1990), 195-218.

The Graduate School of Science and Technology, Kobe University, Rokko-dai 1-1, NADA-KU, KOBE 657-8501, JAPAN

E-mail address: brendle@kurt.scitec.kobe-u.ac.jp 